Proverbio, M., D. G. Vernay, and I. F. C. Smith. "Population-based structural identification for reserve-capacity assessment of existing bridges". Journal of Civil

Structural Health Monitoring, 42369. 2018

\title{
Population-based structural identification for reserve-capacity assessment of existing bridges
}

\author{
Marco Proverbio • Didier G. Vernay • \\ Ian F.C. Smith
}

This is a post-peer-review, pre-copyedit version of an article published in the Journal of Civil Structural Health Monitoring. The final authenticated version is available online at: http://dx.doi.org/10.1007/s13349-018-0283-6

\begin{abstract}
Transportation networks provide an essential contribution to addressing the needs of reliable and safe mobility in urban environments. The core of these networks is made up of infrastructure such as roads and bridges that often, have not been designed to meet current needs. Optimal management requires an accurate knowledge of how existing structures behave. This helps avoid unnecessary replacement and expensive interventions when cheaper and more sustainable alternatives are available. Structural-model updating takes advantage of measurements and more qualitative observations to identify suitable behaviour model classes and values for parameters that influence real behaviour. Error domain model falsification (EDMF) has been proposed as a robust population-based methodology to identify sets of models by comparing finite-element model predictions with measurements at sensor locations. This paper introduces a methodology, which is compatible with EDMF, to assess the reserve capacity of bridges for serviceability and ultimate limit states. A case study the structural identification of a r einforced-concrete bridge in S ingapore - i llustrates the framework developed for the estimation of reserve capacity. Several analyses with increasing levels of model detail using design and updated values of relevant parameters are presented. Traffic-load specifications of design-stage codes (B ritish Code - 1978) and current codes (Eurocodes) are compared. Results show that typical conservative practices carried out during design and construction have led to an as-built reserve capacity of $60 \%$. A large proportion of the as-built reserve capacity has been exploited to accommodate dramatically increased values of traffic-load specifications that are provided by current Singapore codes which have caused a reduction in reserve capacity to $20 \%$. Such a reduction may be less significant in countries where code specifications have not changed as much. Finally, it is shown that advanced methods of analysis and assessment are more suitable than designstage approaches to quantify the reserve capacity.
\end{abstract}

M. Proverbio · D.G. Vernay

ETH Zurich, Future Cities Laboratory, Singapore-ETH Centre, 1 CREATE Way, 138602 Singapore

E-mail: marco.proverbio@epfl.ch.

I.F.C. Smith

Swiss Federal Institute of Technology Lausanne (EPFL), Applied Computing and Mechanics Laboratory (IMAC), School of Architecture, Civil and Environmental Engineering (ENAC), Lausanne, Switzerland Email: ian.smith@epfl.ch. 
Keywords Existing bridges $\cdot$ reserve capacity $\cdot$ structural identification $\cdot$ finite-element model updating $\cdot$ EDMF

\section{Introduction}

A recent report by the World Economic Forum [53] contains the observation that in 2014, world demand for civil infrastructure exceeded supply by one trillion dollars per year. It is not likely that in the future, demand will reduce and it is equally unlikely that supply will be boosted by increased spending on new infrastructure. This situation has led many countries to study alternatives to replacing existing infrastructure. For instance, a recent report [49] estimates the backlog of rehabilitation projects for existing bridges in the USA at $\$ 123$ billion. This trend is further supported by increased recognition of life-cycle costs, embodied energy, sustainability and infrastructure resilience in decision making [17,25].

Fortunately, perceived risks at the design stage, before construction, have led to the introduction of much reserve capacity in civil infrastructure. This reserve often greatly exceeds the target levels of reliability that are introduced by application of safety factors that are stipulated in design codes. However, reserve capacities have not been quantified for the majority of civil structures. Such quantification is complicated by changing service loading, critical limit states involving non-linear behaviour, ageing processes and new traffic requirements that lead to studies of measures such as widening bridges.

Defining the real behaviour of infrastructure through the use of sensing has much potential to provide significant savings when competing options such as replacement, retrofit and improvement are available. Also, when the most attractive options are activities such as retrofit, knowledge of real behaviour results in cheaper and less onerous interventions. Asset managers have much to gain through a more scientific foundation for decisions related to the allocation of resources. However, many challenges remain.

Perhaps the most fundamental challenge is that sensors on infrastructure usually measure effects, not causes. Physics-based models are needed to link effects to causes. Modelfree signal-analysis approaches, for example [30,12] are not useful in such situations. Although these techniques may be appropriate for damage detection [10], they provide weak support for reserve-capacity estimation, and they are of no use for decision-making among alternative scenarios [46].

Design-stage behaviour models need first and foremost to be safe - more than accurate and precise. This means that there are high levels of uncertainty and many sources of systematic bias. Also, the uncertainties at sensor locations in a structural system are correlated, and the value of correlations are influenced by the magnitude of the systematic uncertainty. Once a structure is built, the estimation of reserve capacity for asset management should involve more enhanced models than those used at the design stage. These models need to be capable of representing aspects such as as-built geometry, in-situ boundary conditions, deterioration and real material behaviour.

Since design and existing-structure evaluations are tasks that are performed with various goals in a range of contexts, assessment of structures has been formalised specifically in some codes [47,51,9]. A review of several international codes for assessment of existing bridges - updated to 2012 - is available in [52]. The ISO standard 2394:2015 suggests three approaches (i.e. risk-based, reliability-based and semi-probabilistic) to performance-based decision making. However, most current codes give a full operational elaboration only to semi-probabilistic approaches, while alternative methods may be applied when relevant information is available [23]. A review of reliability-based performance criteria that have been 
used to develop current codes for design and assessment can be found in [18]. For example, in the USA, information of bridge conditions from inspection is incorporated into the load and resistance factor rating for the assessment of bridges [27]. These activities indicate a growing recognition that advanced methods of analysis and assessment help activate reserve capacity that remains hidden when conservative design-like approaches are performed.

Structural identification is used to improve knowledge of structural behaviour [11]. When structures are modelled, for instance using finite elements (FE), structural identification is often referred to as model updating. In model updating, measurements are carried out and used to improve the accuracy of model predictions. While measurements provide additional information for assessment of structures, structural identification involves the use of models that are based on many assumptions and several sources of important uncertainties. Most approaches, such as traditional applications of Bayesian model updating, involve the assumption that uncertainties have zero means [7, 19,24]. When a systematic uncertainty is admitted, it is usually treated as a parameter to be identified, assuming that the systematicbias value is the same at all measurement locations [45]. Furthermore, additional assumptions of uncertainty independence and Gaussian forms are not compatible with the context of civil infrastructure [34]. Error-domain model falsification (EDMF) [21] is a more robust approach to structural model updating compared with traditional Bayesian updating since EDMF provides more accurate (albeit less precise) model-parameter identification without having to make assumptions on values of uncertainty correlations [33].

In order to improve the assessment, advanced models and sophisticated analyses can be conducted. This procedure is similar to the levels-of-approximation methodology [29] used in many codes - that requires further refinements of models if simpler ones are not satisfactory [38]. Much literature, for example [48, 16] includes evaluations of the strength of deteriorated structures by adding into the analysis deterioration mechanisms and their consequences such as reduction of steel diameter, loss of concrete cover and reduction of stiffness due to cracking. Also, two studies [26, 40] involving destructive tests on full-scale bridges highlight the importance of non-linear analyses, material degradation and in-situ boundary conditions for ultimate capacity assessment.

Non-linear finite element analyses (NLFEA) and model calibration have been employed to assess the structural safety of concrete bridges [36, 54]. Use of NLFEA requires the initial setting of several parameters related to characteristics such as constitutive laws of materials that are seldom known precisely and often calibrated through available databases [37], indirect experiments and laboratory tests [13], which are not directly related to the real behaviour of a given structure. Also, model-validation procedures based on minimisation of residuals between expected and observed behaviour may lead to deceptive conclusions, since incorrect assumptions in modelling and wrong values of input parameters can compensate each other thus creating situations where bad model predictions fit observations [33]. Therefore, iterative refinement of FE models [42,35] and strong validation methods based on extrapolation are preferred [46].

In previous studies, the employment of EDMF has led to an increase of $30 \%$ of reserve capacity for deflection on the Langensand Bridge [20]. In that paper, the reserve capacity is defined as the relative difference between the most critical prediction provided by the updated models with the prediction of a design model. No detail on how model uncertainty affects the estimation of reserve capacity is provided. Pasquier et al. [31,32] developed an approach based on EDMF to evaluate the fatigue reserve capacity using the traffic loading obtained by codes and weigh-in-motion measurements. Although this approach integrates model uncertainty into remaining-fatigue-life predictions, the capacity of the structure is assessed by applying partial safety factors and determining the shortest confidence interval 
that includes a probability of $95 \%$ for each prediction location. The most conservative (i.e. the lowest) bound of this interval is the estimated fatigue life. Few probabilistic approaches have been applied to integrate measurements into assessments of the load carrying capacity of existing bridges. Furthermore, no general framework is available to convert structural identification results into serviceability and ultimate capacity assessments.

This paper proposes a data-interpretation approach to assess the reserve capacity (RCap) of existing bridges. This approach covers serviceability and ultimate limit states and complies with current code guidelines for assessment of existing structures. Moreover, the proposed framework is appropriate when population-based methodologies such as EDMF are employed for structural identification. In this approach, deterministic design-values of actions - taken for current codes along with relevant safety factors - are employed, while structural capacity is computed using standard reliability methods. This helps compare results with code requirements.

Previous research involving EDMF has not involved the challenge of ultimate-strength identification behaviour - with the only exception of fatigue since measurement data, which are used for falsification, have been obtained for situations involving elastic behaviour only. This paper provides a strategy to meet this challenge and highlights the importance of identification and advanced simulation for the assessment of ultimate capacity.

First, essential background information on structural reliability and EDMF is presented. Section 3 contains the proposed framework for RCap assessment. Section 4 contains results of a full-scale case study of a bridge. Finally, limitations of alternative analysis and modelling approaches for RCap assessment are discussed.

\section{Background}

\subsection{Reliability analysis for existing structures}

The most general formulation of structural safety in civil engineering involves two stochastic quantities: one, representing the effect of actions (E); the other, a corresponding capacity or resistance $(\mathrm{R})$. Structural safety is verified when:

$$
R \geq E
$$

Traditional checking for structural safety follows deterministic patterns, in which design values of action effects $\left(E_{d}\right)$ and resistance $\left(R_{d}\right)$ are derived from a number of characteristic values by means of safety factors $(\gamma)$. Resistance safety factors consider material properties and model uncertainties. Action safety factors take into account uncertainties in load magnitudes and the type of structural analysis that is conducted. In probabilistic approaches the quantities that influence the problem are introduced as variables, with their distribution types and respective parameters; therefore, no safety factors are required.

Although safety factors guarantee a certain structural safety at design stages, they have not been calibrated for being used in advanced analysis and for assessment purposes. For example, when NLFEA are conducted, some authors suggest considering higher modelling uncertainties than those included in design safety factors [51 43]. Probabilistic approaches help increase the safety-checking accuracy, through including information on action effects and resistance collected during the structural appraisal. Therefore, their employment in RCap estimation is of interest. 


\subsubsection{Probabilistic approaches}

Safety and serviceability are the two principal design criteria for structures. Both requirements are related to a defined period and are traditionally satisfied by minimum-cost strategies. Each requirement is formulated using a limit-state function. The simplest limit-state function is defined as the safety margin which is the difference between action effects and resistance:

$$
G(\boldsymbol{X})=R-E
$$

where $\boldsymbol{X}$ represents the random variables that describe the problem and the assessment requirements.

The failure condition can be expressed in terms of probability of failure:

$$
p_{f}=\operatorname{Prob}[G(\boldsymbol{X})<0]
$$

Although the limit state function often involves many variables and the direct calculation of $p_{f}$ is not possible, there are several methods to compute the probability of failure. In full-probabilistic methods, limit-state functions and distribution functions are introduced with no approximations and calculations are often carried out using stochastic simulations such as the Monte Carlo method. Alternatively, the calculation effort can be reduced though employing approximation methods - usually FORM (First-Order Reliability Method) or SORM (Second-Order Reliability Method). In semi-probabilistic approaches the variables $\boldsymbol{X}$ are introduced by a unique value (i.e. the design value). These methods do not calculate a failure probability, since they only check whether a defined target level is satisfied or not [44].

Considering the limit-state function in Equation (2), it is convenient to measure structural safety in terms of the reliability index $(\beta)$, which is related to the probability of failure:

$$
p_{f}=\Phi(-\beta)
$$

where $\Phi$ is the standard normal probability density function.

If the variables $\mathrm{R}$ and $\mathrm{E}$ are normal or log-normally distributed, the reliability index can be computed directly. Otherwise, the FORM/SORM method may be employed.

\subsubsection{Semi-probabilistic approaches}

In most practical applications, simple semi-probabilistic methods suffice to perform the safety evaluation of a structure. In this approach, the requirement is $\beta \geq \beta_{0}$, whereby $\beta_{0}$ is the safety level prescribed by codes. The design values of resistance $\left(R_{d}\right)$ and action effects $\left(E_{d}\right)-$ described by their mean values $\left(\mu_{R}, \mu_{E}\right)$ and coefficients of variation $\left(\nu_{R}, \nu_{E}\right)-$ are computed, and structural safety is verified when:

$$
R_{d}=\mu_{R} \cdot\left(1-\alpha_{R} \beta_{0} \nu_{R}\right) \geq E_{d}=\mu_{E} \cdot\left(1+\alpha_{E} \beta_{0} \nu_{E}\right)
$$

The expressions $\left(1 \pm \alpha_{x} \beta_{0} \nu_{x}\right)$ are design safety factors, with $\alpha_{R}$ and $\alpha_{E}$ being the socalled sensitivity factors, expressing the importance of each variable in the determination of the probability of failure. It is generally conservative to assume the sensitivity factors $\alpha_{R}=0.8$ and $\alpha_{E}=0.7$. 
Table 1 Reliability classification according to EN 1990

\begin{tabular}{|c|c|c|c|c|}
\hline \multirow[t]{2}{*}{ Reliability classes } & \multirow[t]{2}{*}{ Consequences of failure } & \multicolumn{2}{|c|}{ Target reliability index $\beta_{0}$} & \multirow[t]{2}{*}{ Examples of structures } \\
\hline & & 1 year & 50 years & \\
\hline RC3 & High & 5.2 & 4.3 & Public buildings \\
\hline $\mathrm{RC} 2$ & Medium & 4.7 & 3.8 & Residences/Offices \\
\hline $\mathrm{RC} 1$ & Low & 4.2 & 3.3 & Agricultural buildings \\
\hline
\end{tabular}

\subsubsection{Global resistance safety factor}

One of the simplest stochastic models for the resistance considers $R$ as the product of the nominal resistance $R_{n}$ by the random variables $M, G$ and $F$ [43]:

$$
R=R_{n} \cdot M \cdot G \cdot F
$$

where $M, G, F$ are random variables that represent modelling, geometrical and material uncertainties respectively. Assuming that the bias (systematic variation) is neglected, mean values of those variables are assumed equal to unity (i.e. $\mu_{M}=\mu_{G}=\mu_{F}=1.0$ ). The coefficient of variation is given by:

$$
\nu_{R}=\sqrt{\nu_{M}^{2}+\nu_{G}^{2}+\nu_{F}^{2}}
$$

where $\nu_{M}, \nu_{G}$ and $\nu_{F}$ being the coefficients of variation of $M, G$ and $F$, respectively. It is assumed in the expression above that these variables are statistically independent. However, the evaluation of uncertainty magnitudes is often challenging. While $\nu_{M}$ and $\nu_{G}$ are often taken from the literature, $\nu_{F}$ can be estimated using the methodology initially proposed in [15] and later adopted by the fib Model Code 2010. In this method, the random variation of material properties is assessed using only two non-linear analyses: one using mean material properties $\left(R_{m}\right)$, and one using characteristic properties $\left(R_{k}\right)$ - these characteristics are established according to statistical data as described in [3] . Then, the coefficient of variation of material uncertainty $\left(\nu_{F}\right)$ is computed according to the following equation:

$$
\nu_{F}=\frac{1}{1.65} \cdot \ln \left[\frac{R_{m}}{R_{k}}\right]
$$

Based on the assumption of a log-normal distribution for resistance, which is often proposed in codes, and using the notation introduced above, the global resistance safety factor $\gamma_{R}$ is given by:

$$
\gamma_{R}=\exp \left(\alpha_{R} \cdot \beta_{0} \cdot \nu_{R}\right)
$$

\subsubsection{Target reliability index}

Target reliability levels required in structural codes represents the trade-off between cost optimization, risk management and quality control. In EN 1990 [3] the target reliability index $\left(\beta_{0}\right)$ is given for two reference periods (1 year and 50 years). These values are related, for each reliability class, to the same reliability level. However, no explicit link between the target reliability level and design working life is provided. The values reported in Table 1 refer to the ultimate limit state (ULS). However, target $\beta$ can be defined for irreversible serviceability limit states (SLS). For example, for a class RC2 structural member, $\beta_{0}$ for the SLS are equal to 2.9 for 1 year and 1.5 for 50 years [3]. Alternative values are suggested in [51]. 


\subsection{Model falsification for structural identification}

Error-domain model falsification (EDMF) - initially proposed in probabilistic terms in [21] - helps identify plausible physics-based models using information provided by measurement data. Plausible models are defined by parameter values that are instances of a model class. Each model class has a unique parameterization that includes characteristics such as material properties, geometry, boundary conditions and actions.

Let $n_{y}$ be the number of measurement locations. For each location, $i \in\left\{1, \ldots, n_{y}\right\}$, $\widehat{Y}_{i}$ denotes the real response of a structure (unknown in practice) and $y_{i}$ corresponds to the measured value at location $i$. Predictions $g_{k}\left(x_{i}, \boldsymbol{\theta}\right)$ are evaluated at location $x_{i}$ through assigning a vector of parameter values $\boldsymbol{\theta}$ to the selected model class $g_{k}$. Evaluation of predictions is usually carried out using FE analyses. Since model-prediction uncertainty $U_{i, g_{k}}$ and measurement uncertainty $U_{i, y}$ are unavoidable, model predictions and measurements are linked to the real behavior using the following equation:

$$
g_{k}\left(x_{i}, \boldsymbol{\theta}\right)+U_{i, g_{k}}=\widehat{Y}_{i}=y_{i}+U_{i, y} \quad \forall i \in\left\{1, \ldots, n_{y}\right\}
$$

Rearranging the terms and combining the difference between the two sources of uncertainty $U_{i, g}$ and $U_{i, y}$ in a unique source and $U_{i, c}$, we obtain:

$$
g_{k}\left(x_{i}, \boldsymbol{\theta}\right)-y_{i}=U_{i, c}
$$

The left-hand side of Equation (11) is the difference between a model prediction and a measurement at location $\mathrm{i}$, which is often called the residual $r_{i}=g_{k}\left(x_{i}, \boldsymbol{\theta}\right)-y_{i}$.

The uncertainty related to measurements $U_{y}$ is usually estimated by conducting multiple series of tests under site conditions. Manufacturer specifications are often optimistic lower bounds on the in-situ uncertainty. The uncertainty related to the model class $\left.U_{(} g_{k}\right)$ is estimated using values taken from the literature, stochastic methods (to estimate uncertainties of parameters that are not included in the model-class parametrization), engineering judgment and local knowledge. In real situations, uncertainties associated with the model class are usually much larger than measurement uncertainties.

EDMF selects plausible behaviour models by falsifying those for which residuals exceed thresholds that are defined in the uncertainty domain (i.e., the error domain).This facilitates combination of uncertainties related to parameters with model uncertainty. First, an initial model set is generated by assigning parameter values to the model class using sampling techniques. Then, threshold bounds are defined at each sensor location, according to a confidence level fixed at $95 \%$ - common practice in many engineering problems. Models for which residuals are within threshold bounds at each sensor location are included in the candidate model set (CMS). Models for which residuals exceed these bounds are falsified.

When a candidate model set is identified, prediction tasks involve using the models in the CMS to assess the reserve capacity of the structure. Predictions $Q_{j}$ at $n_{q}$ locations are given by:

$$
Q_{j}=g_{k}\left(x_{i}, \boldsymbol{\theta}^{\prime \prime}\right)+U_{i, g_{k}}, \quad \forall i \in\left\{1, \ldots, n_{q}\right\}
$$

Where $\boldsymbol{\theta}^{\prime \prime}$ is a set of combinations of parameter values representing the CMS and $U_{g_{k}}$ is the model uncertainty. The performance of identification in reducing the initial parameter uncertainties depends on factors such as the initial choice of parameters, the sampling technique and the sensor configuration.

When all initial model instances generated are falsified, the entire model class is falsified. This means that no model is compatible with observations given model and measurement uncertainties. Thus it is usually a sign of incorrect assumptions in the model-class 
definition. In such situations, practising engineers should i) review initial assumptions on the adopted model class $g_{k}$ - for example, through collecting additional information in situ - ii) evaluate whether or not uncertainties should be increased and iii) check for the presence of outliers in measurements. An EDMF-compatible iterative framework for data interpretation and its application for performance assessments is described in [35].

\section{Population-based reserve-capacity assessment}

This paper provides a data-interpretation methodology that is compatible with EDMF to assess the reserve capacity of existing structures. The general framework is depicted in Figure 1 Initially, EDMF is used to identify the CMS. Then, the reserve capacity is assessed either through computing the CMS failure probability - when the SLS is investigated - or using the global resistance safety factor - when the ULS is investigated. The term failure indicates a condition for which structural safety is not verified (see Equation 1). Such a condition corresponds to the structural collapse for the ULS and the infringement of code requirements for the SLS.

Since reserve-capacity estimation requires the increment of applied loads until a limit state is reached, several simulations must be computed for each candidate model. In the elastic behaviour (i.e. for the SLS), the linear relationship between load magnitude and model predictions allows direct determination of predictions under increasing loads. When the ultimate RCap is investigated, predictions should be computed using FE solvers for each candidate model and each load step until failure using non-linear finite element analyses (NLFEA). However, NLFEA are often computationally demanding and time-consuming. Therefore, a probabilistic approach, in which all candidate models are used for predictions, is appropriate for the SLS reserve-capacity estimation while a global safety factor approach, in which a small subset of candidate models is taken into account, is suitable for the ULS.

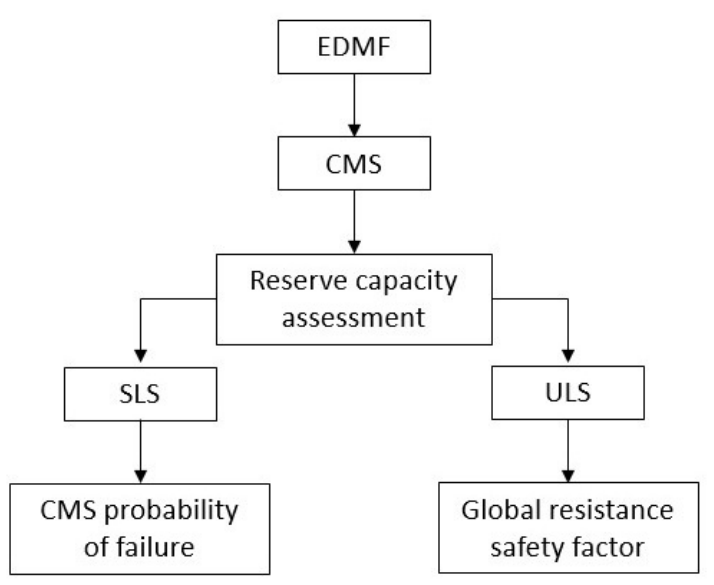

Fig. 1 General framework for reserve-capacity assessment 


\subsection{Serviceability limit states - SLS}

The proposed probabilistic approach to assess the serviceability-limit-state reserve capacity $\operatorname{RCap}_{S L S}$ is depicted in Figure 2

First, the test load is applied to the FE model class and parameter values, for which model predictions are compatible with measurements, are identified using EDMF. Then, the code design loads - which include all load safety factors for the SLS - are assigned to the CMS and model predictions $g_{S L S}\left(x_{j}, \boldsymbol{\theta}^{\prime \prime}\right)$ are computed for each candidate model. According to Equation (11, model uncertainty $U_{j, g_{S L S}}$ and model predictions $g_{S L S}\left(x_{j}, \boldsymbol{\theta}^{\prime \prime}\right)$ are combined using the Monte Carlo method. As a result, the distribution of predictions $Q_{j, g_{S L S}}$, which include model uncertainty, is obtained and the failure probability $P_{f}^{C M S}$ can be computed.

In order to estimate the RCap, $P_{f}^{C M S}$ is compared with the SLS target failure probability $P_{f}^{S L S}$ - typical values are described in Section 2.1.4 As long as the target failure probability is greater than the CMS failure probability, design loads are increased using a load factor $(L F>1)$, which is applied to the critical variable design load (e.g. the traffic load). When the CMS failure probability becomes equal to the target probability $P_{f}^{S L S}$ the limit state is reached and the value of load factor $\left(L F_{S L S}\right)$ is taken to be the reserve capacity of the structure. Since the LF is applied to the design value of critical variable load, the SLS RCap represents the extra carrying capacity beyond design safety factors.

If the CMS failure probability under design loads $(L F=1)$ is greater than $P_{f}^{S L S}$ no SLS RCap is found. In this situation, further investigation is needed in order to improve the initial assumptions on parameter intervals and model uncertainty. Also, weight-in-motion data may be used to replace code traffic-load specifications with probabilistic traffic-load distributions.

\subsection{Ultimate limit states - ULS}

The proposed probabilistic approach to assess the ultimate-limit-state reserve capacity $R C a p_{U L S}$ is depicted in Figure 3 .

Traditional applications of EDMF employ elastic measurements to update parameters of FE model classes. When the ultimate behaviour is instigated, the model class should be modified to consider only elements that are relevant to failure. For example, parameters related to geometrical properties such as the thickness of a bridge deck are relevant at the ULS - these parameters are referred to as active parameters in this paper. On the contrary, boundary conditions (i.e. stiffness of bearing devices) identified in the elastic domain are likely to be unrealistic at failure - these parameters are referred to as inactive parameters. Values of active parameters are assigned to the ULS model class, $g_{U L S}$, while values of other parameters are defined according to design assumptions.

Mean values $\boldsymbol{\theta}_{\boldsymbol{m}}$ and characteristic values $\boldsymbol{\theta}_{\boldsymbol{k}}$ of each active parameter are computed using the CMS and two models $\left(g_{U L S}\left(\boldsymbol{\theta}_{\boldsymbol{m}}\right)\right.$ and $\left.g_{U L S}\left(\boldsymbol{\theta}_{\boldsymbol{k}}\right)\right)$ are generated through assigning these values to the ULS model class. The values $\theta_{m}$ and $\theta_{k}$ are calculated using the discrete parameter distributions provided by the CMS. Characteristic values are defined as the 5\% or $95 \%$ fractiles using the least favourable value for the current analysis of CMS parameter distributions. Then, $g_{U L S}\left(\boldsymbol{\theta}_{\boldsymbol{m}}\right)$ and $g_{U L S}\left(\boldsymbol{\theta}_{\boldsymbol{k}}\right)$ are solved using non-linear analyses in which the design loads are increased, using a load factor $(L F>1)$, until failure. Load factors for the ULS are applied to the global vertical load of the bridge (i.e. sum of self-weight, dead loads and variable loads). Compared with linear analyses, NLFEA involves higher 


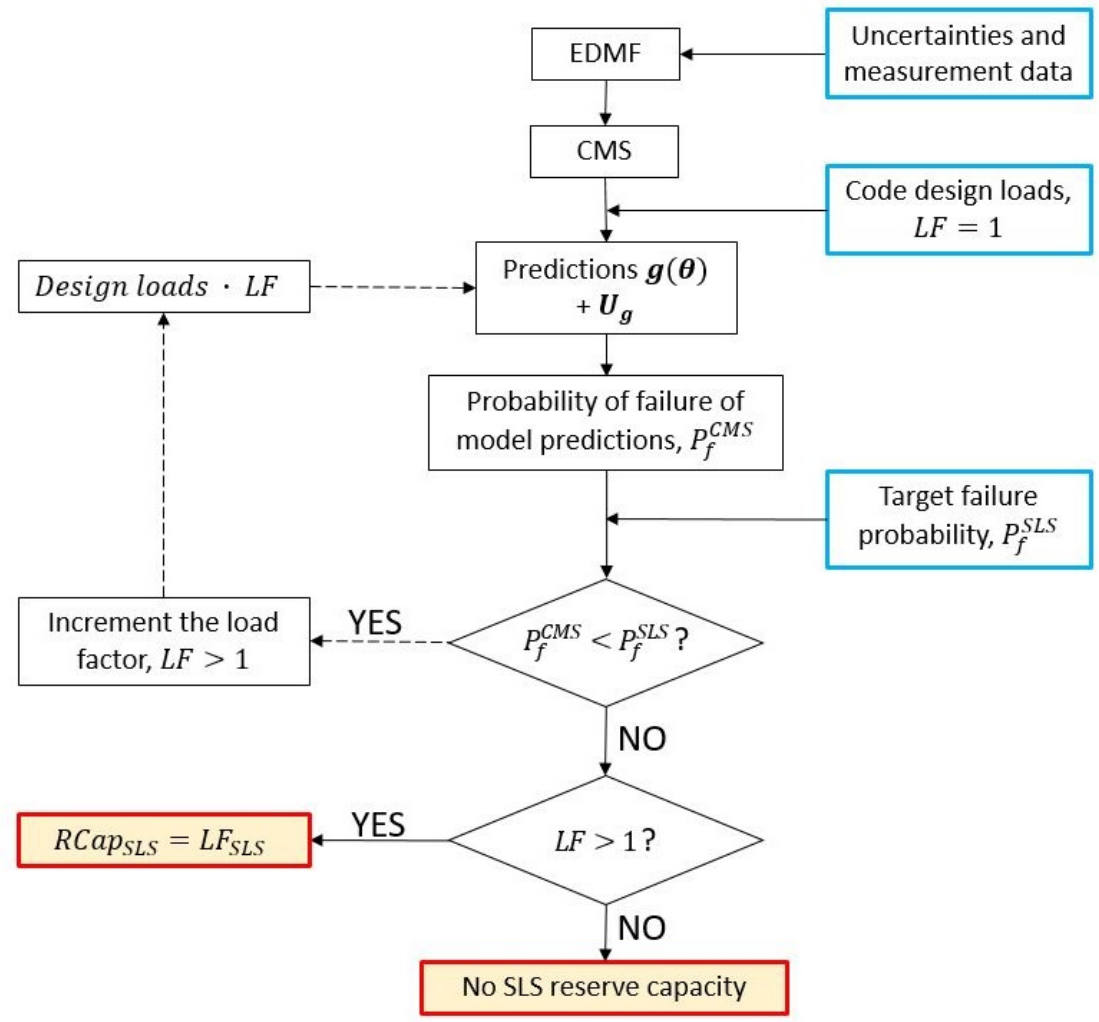

Fig. 2 Flow chart for SLS reserve-capacity assessment. Blue boxes represent input data, while red boxes contain the outputs. The dashed arrows indicate a subpath that is carried out until the SLS is reached.

uncertainties to account for approximations in geometry, material and modelling. These uncertainties are combined and included in the global resistance safety factor $\gamma_{R}$. Values of the load factor at failure, which are referred to as $L F_{m}$ and $L F_{k}$, are used to estimate the material uncertainty. Finally, $\gamma_{R}$ is computed according to Equation 9]. The RCapULS is computed as the ratio between the load factor at failure using mean parameter values $\left(L F_{m}\right)$ and the global safety factor $\left(\gamma_{R}\right)$.

Since the ULS reserve-capacity framework requires only two non-linear simulations, computation times can be reduced. In particular, advantages arise when advanced FE models, which often require long computation times, are employed.

In Equation (8), $R_{m}$ and $R_{k}$ are computed through increasing design loads until failure by means of load factors. Using the symbols adopted in the EN 1990, this can be written as follows:

$$
\frac{R_{m}}{R_{k}}=\frac{L F_{m} \cdot\left(\gamma_{g} G_{k}+P+\gamma_{Q} \overline{Q_{k}}\right)}{L F_{k} \cdot\left(\gamma_{g} G_{k}+P+\gamma_{Q} \overline{Q_{k}}\right)}=\frac{L F_{m}}{L F_{k}}
$$

where $\overline{Q_{k}}=Q_{k, j}+\sum_{i \neq j}\left(\psi_{0, i} \cdot Q_{k, i}\right)$ is the combination of all variable loads, $G_{k}$ are permanent loads, $P$ is the applied prestress, and $\gamma$ are load safety factors. The term in parentheses is the design load (i.e. the sum of self-weight, permanent and variable loads) that 


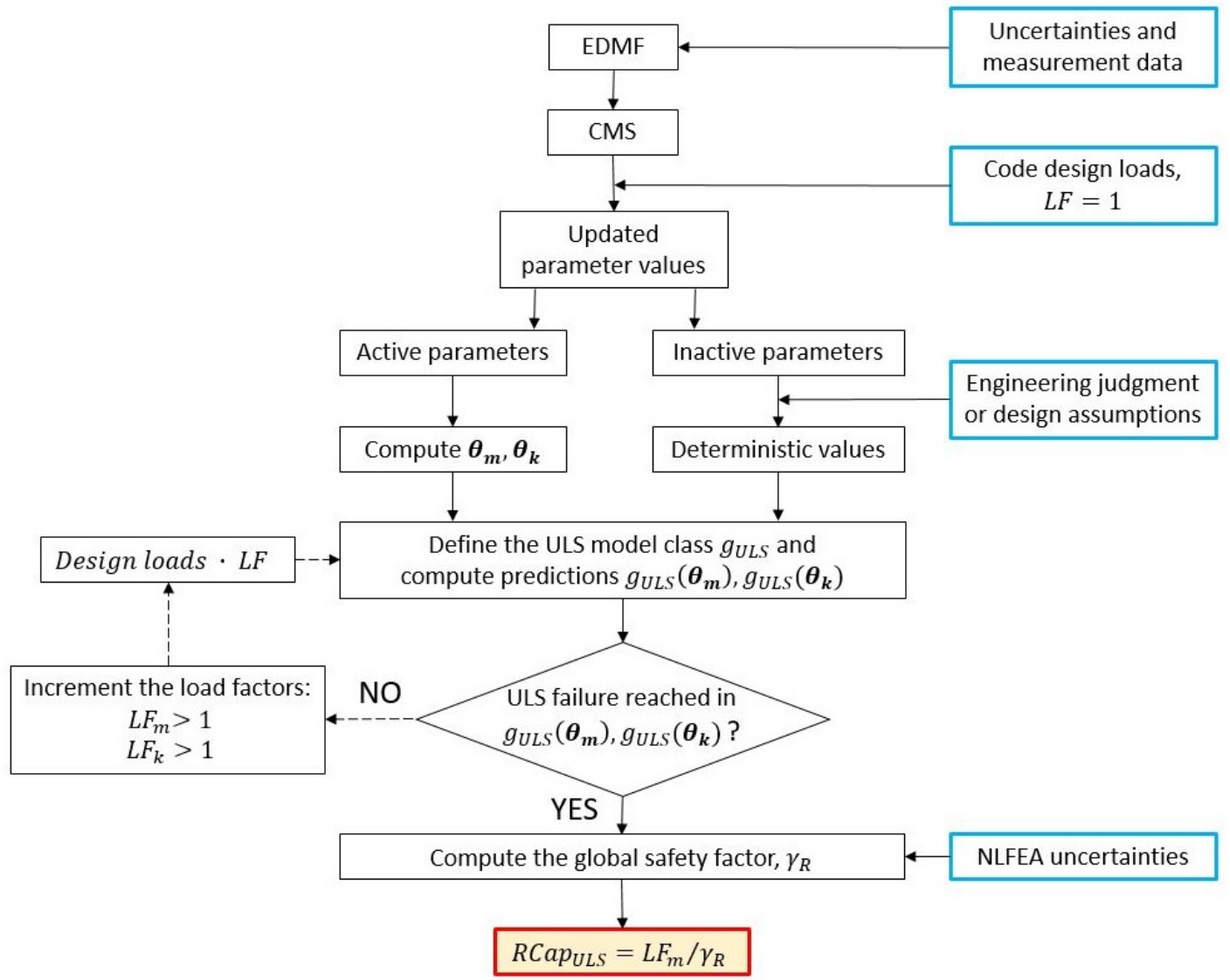

Fig. 3 Flow chart for ULS reserve-capacity assessment. Blue boxes represent input data, while the red box contains the output. The dashed arrows indicate a subpath that is carried out for both $g_{U L S}\left(\boldsymbol{\theta}_{m}\right)$ and $g_{U L S}\left(\boldsymbol{\theta}_{k}\right)$ until failure.

is carried by the structure. In the analyses design loads are constant while both mean and characteristic values of material properties are included.

In Equation (13), load factors $\left(L F_{m}\right.$ and $\left.L F_{k}\right)$ should be applied to the global vertical load of the structure (i.e. the sum of self-weight, permanent and variable loads). Otherwise, the resistance ratio $\left(R_{m} \backslash R_{k}\right)$ cannot be computed directly as the ratio of the two load factors. Therefore, the load factor for the ULS is applied to the global vertical load while the load factor for the SLS is applied only to the critical variable design load.

\section{Case study}

The case study is used to apply the methodologies presented in Section 3 for SLS and ULS reserve-capacity assessment. A range of alternative approaches is presented, and their results are compared.

The flyover, which consists of four prestressed concrete beams, has a single span of $32 \mathrm{~m}$ and is supported at each end by four bearing devices (Figure 4 4 ). The beams support and are 
connected to a reinforced concrete slab that is $22 \mathrm{~cm}$ thick. The FE model class that has been used for falsification also includes non-structural elements such as the asphalt pavement and precast concrete barriers. These elements, which are usually included as permanent loads in design-stage models, are considered to reduce model-simplification uncertainties.

A static load test involving six trucks, each with a gross weight of 33 tons, is performed. The measurement system consists of a laser tracker, eight strain gauges (S) and two inclinometers (I). In order to increase the accuracy of deflection measurements, four prisms (P) are placed on the bottom faces of the main beams. Truck configuration and sensor locations are depicted in Figure 4 a, b.

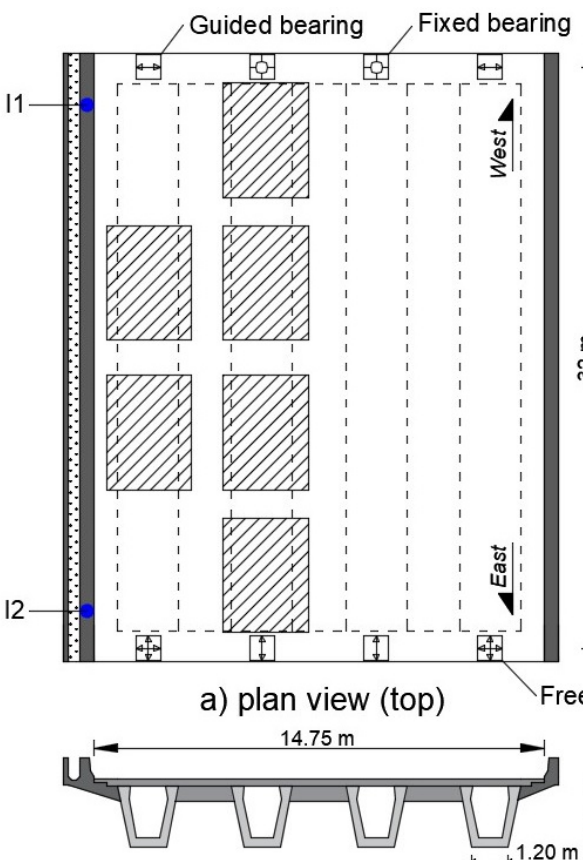

c) cross-section

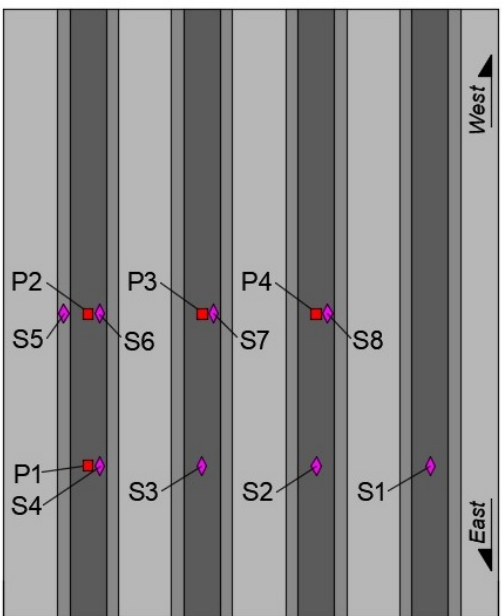

b) bottom view $32.00 \mathrm{~m}$

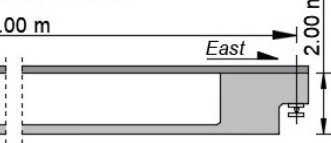

d) elevation

Fig. 4 Flyover plan view (top) a), bottom view b), cross-section c) and elevation d). The truck configuration is shown along with the position of two inclinometers (I), four deflection prisms $(\mathrm{P})$ and eight strain gauges (S).

\subsection{Uncertainty definition}

The parameters are defined according to a sensitivity analysis of parameter impact on model predictions at measurement locations. Parameter selection is often a trade-off. Although additional parameters may provide further insights, the sampling quality decreases when high-dimensional parameter domains are explored. In this study, the five most sensitive parameters are included for identification and an adaptive sampling methodology is used to sample the five-dimensional parameter space defined by the Youngs modulus of cast-inplace concrete, the Youngs modulus of precast concrete, the Youngs modulus of barrier 
concrete, the rotational and the vertical stiffness of the bearing devices. The same approach has been used in [?]. The initial interval for each parameter is reported in Table 2 Table 3 de-

Table 2 Initial intervals of parameters.

\begin{tabular}{lll}
\hline Parameters & Lower bound & Upper bound \\
\hline$\theta_{1}-$ Young's modulus of cast-in-place concrete & $20 \mathrm{GPa}$ & $35 \mathrm{GPa}$ \\
$\theta_{2}$ - Young's modulus of precast concrete & $25 \mathrm{GPa}$ & $50 \mathrm{GPa}$ \\
$\theta_{3}$ - Young's modulus of barrier concrete & $3 \mathrm{GPa}$ & $40 \mathrm{GPa}$ \\
$\theta_{4}$ - Rotational stiffness of bearing devices & $9 \log (\mathrm{Nmm} / \mathrm{rad})$ & $13 \log (\mathrm{Nmm} / \mathrm{rad})$ \\
$\theta_{5}-$ Vertical stiffness of bearing devices & $8 \log (\mathrm{N} / \mathrm{mm})$ & $11 \log (\mathrm{N} / \mathrm{mm})$ \\
\hline
\end{tabular}

scribes modelling and measurement uncertainty sources. Uniform distributions are adopted to describe model uncertainty sources. The minimal and maximal bounds defined in Table 3 are expressed as a percentage of the mean value of model predictions for modelling uncertainties and as a percentage of the measured value or in absolute terms for measurement uncertainties. The uncertainty associated with model simplifications and the FE method has been estimated considering the model class characteristics. This source takes into account that only the most sensitive parameters - according to a sensitivity analysis of the relative importance of each structural parameter in model predictions - have been selected for identification. In the FE model of the bridge adjacent interfaces are perfectly connected. This assumption leads to a model class that is likely to overestimate the real stiffness of the bridge. Therefore, the FE uncertainty distribution is not centred on zero. Finally, the source of uncertainty associated with spatial variability originates from strain sensors measuring behaviour that is affected by the spatial variability of material properties.

Sensor accuracies are based on manufacturer specifications. The measurement repeatability is assessed by taking multiple measurements under the same load case. For strain gauges, an uncertainty also arises from the imperfect alignment of gauges with respect to the bridge longitudinal axis. Finally, additional noise associated with sensor installation have been considered for inclinometers and strain gauges using engineering judgment.

More detail of the uncertainties adopted in this case study and further references can be found in [?:50].

Table 3 Modelling and measurement uncertainty sources.

\begin{tabular}{lllllll}
\hline Uncertainty source & \multicolumn{2}{c}{ Displacements - (P) } & \multicolumn{2}{c}{ Rotations - (I) } & \multicolumn{2}{c}{ Strains - (S) } \\
\hline & Min & Max & Min & Max & Min & Max \\
\hline Model simplifications and FE method (\%) & -5 & 13 & -5 & 13 & -5 & 13 \\
Mesh refinement (\%) & -1 & 1 & -1 & 1 & -1 & 1 \\
Spatial variability (\%) & - & - & - & - & -5 & 5 \\
Additional uncertainty (\%) & -1 & 1 & -1 & 1 & -1 & 1 \\
\hline Sensor accuracy & $-0.05 \mathrm{~mm}$ & $0.05 \mathrm{~mm}$ & $-1 \mu \mathrm{rad}$ & $1 \mu \mathrm{rad}$ & $-2 \mu \epsilon$ & $2 \mu \epsilon$ \\
Repeatability & $-0.15 \mathrm{~mm}$ & $0.15 \mathrm{~mm}$ & $-4 \mu \mathrm{rad}$ & $4 \mu \mathrm{rad}$ & $-4 \mu \epsilon$ & $4 \mu \epsilon$ \\
Sensor orientation (\%) & - & - & - & - & 0 & 6 \\
Sensor installation (\%) & - & - & -5 & 5 & 0 & 5 \\
\hline
\end{tabular}




\subsection{Model Falsification and CMS}

In order to perform model falsification, for each measurement location, a combined uncertainty is computed, and threshold bounds are determined for a confidence level fixed at $95 \%$.

Figure 5 shows the results of structural identification. The vertical axes represent parameter values $\left(\theta_{i}\right)$ and predictions at sensor locations, which are referenced in accordance with Figure 4 Each grey line represents an instance of the initial model set along with the predictions of this instance at each sensor location. Models for which residuals are within threshold bounds at each sensor location are included in the CMS (red lines). The reduc-

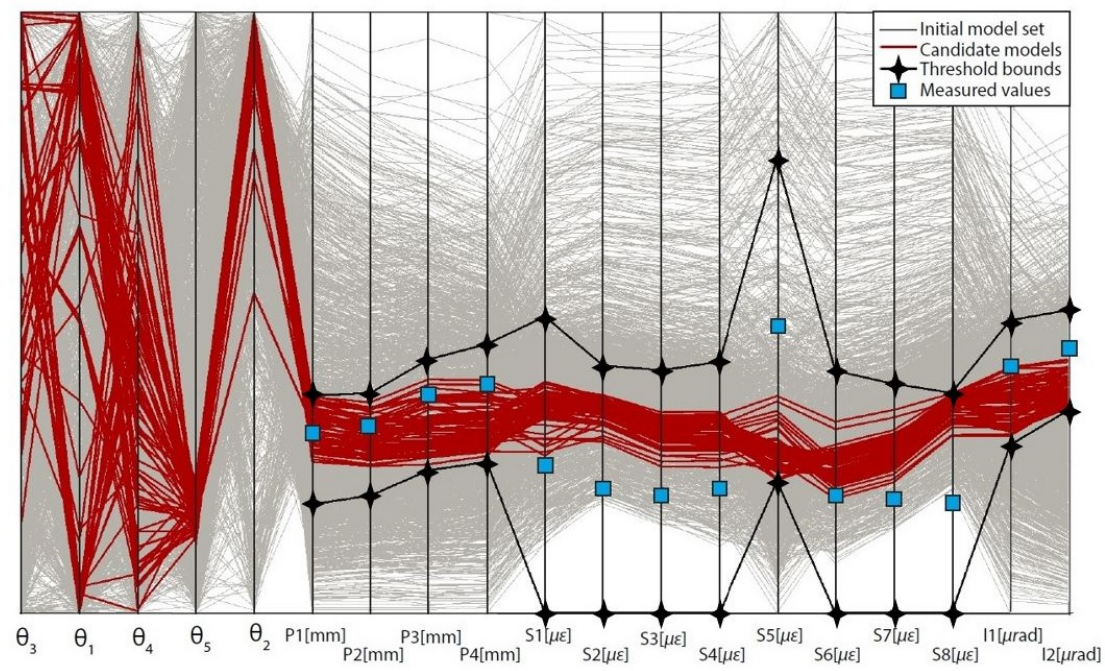

Fig. 5 Parallel axis plot of structural identification using EDMF

tion of parameter ranges after falsification is related to the measurement system adopted. Some parameters such as the vertical stiffness $\left(\theta_{5}\right)$ and the Youngs modulus of precast concrete $\left(\theta_{2}\right)$ are well identified, while for other parameters such as the Youngs modulus of site-cast concrete $\left(\theta_{1}\right)$, falsification does not reduce the initial interval. The performance of identification in reducing the initial parameter uncertainties depends on factors such as the initial choice of parameters, the sampling technique and the sensor configuration. Updated parameter intervals are reported in Table 4

Table 4 Identified values of parameters

\begin{tabular}{lc}
\hline Parameters & Identification interval \\
\hline$\theta_{1}$ - Young's modulus of cast-in-place concrete $[\mathrm{GPa}]$ & $20.00-34.98$ \\
$\theta_{2}$ - Young's modulus of precast concrete [GPa] & $38.31-44.99$ \\
$\theta_{3}$ - Young's modulus of barrier concrete $[\mathrm{GPa}]$ & $8.48-39.99$ \\
$\theta_{4}$ - Rotational stiffness of bearing devices $[\log (\mathrm{Nmm} / \mathrm{rad})]$ & $9.02-12.87$ \\
$\theta_{5}$ - Vertical stiffness of bearing devices $[\log (\mathrm{N} / \mathrm{mm})]$ & $8.34-8.67$ \\
\hline
\end{tabular}




\subsection{Reserve capacity for SLS}

The serviceability assessment of existing structures is often conducted using visual inspections and experience of end users. This practice is also accepted in some recent codes [4] if actions remained unchanged from the design stage to the assessment stage. However, when live loads are expected to increase, the serviceability limit state should be verified using current-code requirements [4].

The bridge analysed in this study was designed in the 80s according to the British Codes (BC) [1,2], while the current structural codes adopted in Singapore are the Eurocodes (EC) [5]6]. Figure 6 depicts the SLS traffic load adopted in the BC and the Singapore Annex to EC. Considering a 6-meter-long section across the bridge mid-span, the total EC traffic load $(1,535 \mathrm{kN})$ is $60 \%$ higher than the $\mathrm{BC}$ traffic load $(946 \mathrm{kN})$. Therefore, serviceability requirements should be verified, and updated models can be used to quantify the bridge reserve capacity, to improve the inspection planning and to optimise maintenance activities. The SLS requirements indicated in the SS EN 1992-2 include: stresses limitation, crack

a) BS 5400-2:1978

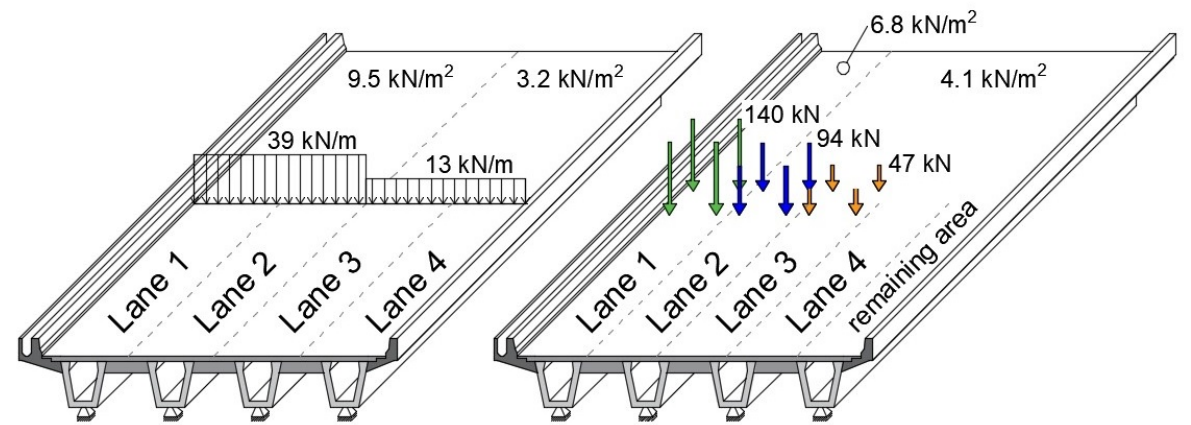

Fig. 6 Serviceability limit state (SLS) traffic loads according to the British Standards 1978 a) and the current Singapore Annex to Eurocode $1 \mathrm{~b}$ ).

and deflection control. For concrete structures corrosion of reinforcement is often the main deterioration process [22]; therefore, crack-control is the critical check to be conducted. More precisely, the EC [6] and the BC [2] require that all concrete within $100 \mathrm{~mm}$ of bonded tendons or their ducts remains in compression under frequent-load combinations. The only difference between the EC and the BC lies in the specification for design traffic loads.

Table 5 reports the SLS analyses that have been performed. Each analysis is carried out using either the EC traffic load or the BC traffic load.

Table 5 Summary of SLS reserve-capacity estimations.

\begin{tabular}{lllll}
\hline & Model / Analysis & Traffic Load & Parameter values & RCap assessment \\
\hline$S L S_{1}$ & No FE model / design equations & EC/BC & Codes & Single model \\
$S L S_{2}$ & 3D FE model / linear & EC/BC & Codes & Single model \\
$S L S_{3}$ & 3D FE model / linear & EC/BC & EDMF & Population based \\
\hline
\end{tabular}

$\mathrm{EC}=$ Eurocodes $[5] ; \mathrm{BC}=$ British codes $[1]$ 


\subsubsection{Single-model methods}

In this section the reserve capacity is assessed using a unique model that is defined by deterministic parameter values, which correspond to the design values.

\subsubsection{Simplified analysis $-S L S_{1}$}

In this simplified analysis, only the most loaded beam of the bridge is studied, and loads are estimated using the area-of-influence method (Figure 7). $S L S_{1}$ represents the design approach, in which parameter values are inferred from codes. Design loads are increased using a load factor $(L F>1)$ until the SLS (i.e. tension in the concrete around bonded tendons) is reached. Load-factor values at the limit state correspond to the SLS reservecapacity estimations.

The $R \operatorname{Cap}_{S L S}$ is equal to 1.53 under the BC traffic-load specifications and 0.98 under the EC traffic-load specifications.

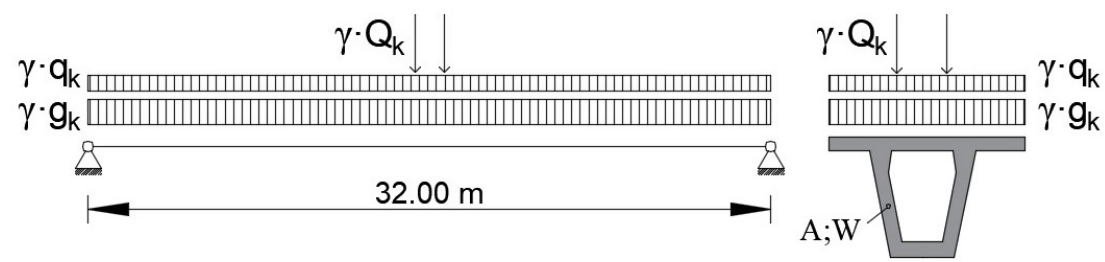

Fig. 7 Simplified model of the bridge most-loaded beam.

4.3.1.2 3D linear analysis $-S L S_{2}$

In $S L S_{2}$ the entire bridge structure is analysed. Parameter values are taken from codes, and the FE model class is based on the design assumptions reported in Table 6. Parameter design values for $\theta_{1}$ and $\theta_{4}$ are included in identification intervals, while identified values of precast-concrete Young-modulus are higher than conservative code values. Moreover, low values of bearing vertical-stiffness are identified compared with the rigid-support assumption that is common for design-stage models.

The $R \operatorname{Cap}_{S L S}$ is equal to 1.82 under the $\mathrm{BC}$ traffic-load specifications and 1.42 under the EC traffic-load specifications. This example shows that advanced FE models often provide higher RCap estimations compared with design-stage simplified analyses.

Table 6 Identified and parameter design values for the SLS. Design assumptions refer to original drawings and the British Codes while design values are taken from the Eurocodes.

\begin{tabular}{lllc}
\hline Parameters & Identification interval & Design assumption & SLS design values \\
\hline$\theta_{1}[\mathrm{GPa}]$ & {$[20.00-34.98]$} & BS 5400-4: grade 30/20 & $\mathrm{E}=31 \mathrm{GPa}$ \\
$\theta_{2}[\mathrm{GPa}]$ & {$[38.31-49.99]$} & BS 5400-4: grade 50/20 & $\mathrm{E}=35 \mathrm{GPa}$ \\
$\theta_{3}[\mathrm{GPa}]$ & {$[8.48-39.99]$} & Barriers are permanent loads & - \\
$\theta_{4}[\log (\mathrm{Nmm} / \mathrm{rad})]$ & {$[9.00-12.87]$} & Perfect hinge - no friction & $9 \log (\mathrm{Nmm} / \mathrm{rad})$ \\
$\theta_{5}[\log (\mathrm{N} / \mathrm{mm})]$ & {$[8.34-8.67]$} & Rigid support - no settlement & $11 \log (\mathrm{N} / \mathrm{mm})$ \\
\hline$E=$ Young's modulus & & &
\end{tabular}




\subsubsection{Population-based method}

In this section, the reserve capacity is assessed using the CMS.

4.3.2.1 3D linear analysis $-S L S_{3}$

In $S L S_{3}$ the model class used for falsification is employed. The methodology to estimate $R_{C a p_{S L S}}$ takes into account the CMS prediction distribution and includes model uncertainties. The number of samples that are taken into account when model uncertainties are added to model predictions is related to the coefficient of variation of the probability of failure $P_{f}$ by the following equation:

$$
\nu_{p_{f}} \approx \frac{1}{\sqrt{z \cdot P_{f}}}
$$

Where $\nu_{p_{f}}$ is the coefficient of variation of $P_{f}$ and $z$ is the number of samples. Considering that standard values of failure probabilities for SLS are $P_{f}^{S L S}=10^{-1}$ (for a 50-year period) and $P_{f}^{S L S}=10^{-3}$ (for a 1-year period), to ensure small coefficient of variation - e.g., $<10 \%$ - as many as $z=10^{4}$ and $z=10^{6}$ samples are required.

Figure 8 shows the application of the framework to assess SLS RCap under the EC traffic-load specifications. First, the CMS prediction distribution is computed (Figure 8p). Then, the model uncertainty (Figure 8p) is added to the CMS stress predictions using the Monte Carlo method. The limit state condition (i.e. tension in concrete around bonded tendons) is represented by the vertical line that corresponds to the null stress (Figure 8c). Since the discrete distribution of stress predictions lies on the compression side of the limit state, the reserve capacity can be assessed by increasing design loads.

Figure 9 shows the discrete distribution of stress predictions when a $L F=1.29$ is applied to design loads. In this situation, the CMS failure probability equals the target probability $P_{f}^{S L S}=0.1$. Therefore, this value of load factor corresponds to the SLS reserve capacity $\left(R \operatorname{Rap}_{S L S}=L F_{S L S}=1.29\right)$.

The same procedure is executed under the BC traffic-load specifications. Results are reported in Table 7

\subsubsection{Summary of SLS results}

Table 7 reports values of $R \operatorname{Cap}_{S L S}$ for the SLS under the BC and the EC. Since BC trafficload specifications for the 80 s are lower than traffic-load specifications prescribed by the Singapore-Annex-to-EC1 traffic-load specifications, there is more reserve capacity when former codes are used.

When conservative design-like analyses $\left(S L S_{1}\right)$ are used for serviceability assessments, no reserve capacity is found under current EC traffic-load specifications $\left(R C a p_{S L S}=\right.$ $0.98)$. On the contrary, significant reserve is observed under the $\mathrm{BC}$ traffic loads (RCap $\operatorname{RLS}_{S}=$ 1.53). This difference is related to increased traffic loads that are specified in the Singapore Annex to EC1.

When simplified models are replaced by 3D FE models $\left(S L S_{2}, S L S_{3}\right)$, higher RCap values are found. However, when parameter design values are employed $\left(S L S_{2}\right)$, the RCap is overestimated compared with the assessment provided by the CMS prediction distribution $\left(S L S_{3}\right)$.

This situation is a consequence of the limit state that is under investigation (i.e. crack control). The bridge precast beams are connected to a cast-on-site deck. The EC design 
a) CMS stress-prediction distribution

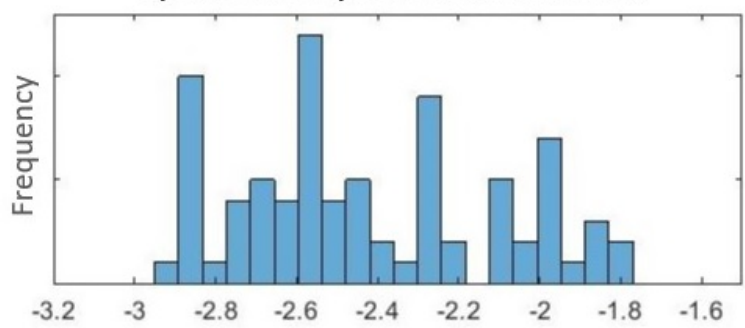

b) Model-uncertainty stress distribution

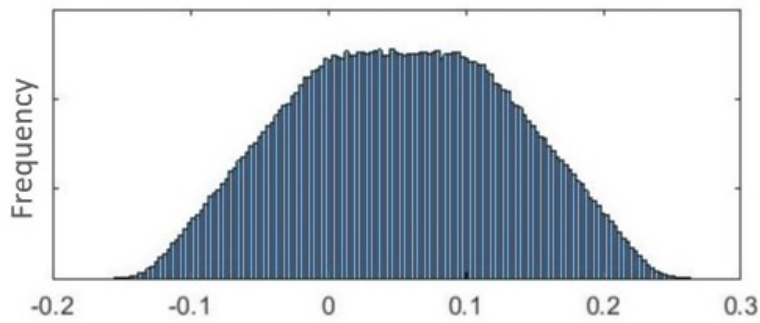

c) Prediction + model-uncertainty distribution

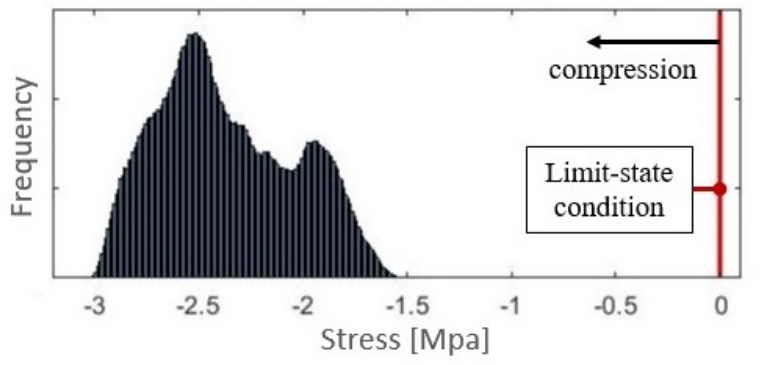

Fig. 8 CMS stress-prediction distribution (a), model-uncertainty stress distribution (b) and their combination (c) under the EC traffic load. The serviceability-limit-state condition is shown using the red vertical line.

assumptions (see Table 6) consist of a unique value of stiffness for the beam cross-section. Several combinations of Youngs modulus values (i.e. deck and precast beams) results in alternative load redistributions, neutral-axis depths and, therefore, stress values on beam cross-sections. Since crack control aims to reduce the tensile stress in concrete around the tendons, models that show high tensile stresses at beam bottom faces are less conservative. Since several combinations of updated values of Youngs modulus - parameters $\theta_{1}$ and $\theta_{2}$ in Table 6- are considered in $S L S_{3}$, the CMS prediction distribution includes stress values close to the limit state. This helps avoid flawed RCap estimations resulting from the wrong selection of the critical parameter combination.

Taking advantage of measurements and advanced simulations the as-built serviceability reserve capacity of the bridge is equal to $64 \%$ - resulting from conservative practices in design and construction. Following changes in traffic-load specifications from the British Code to the Eurocode, the reserve capacity at the SLS went down to $29 \%$. 


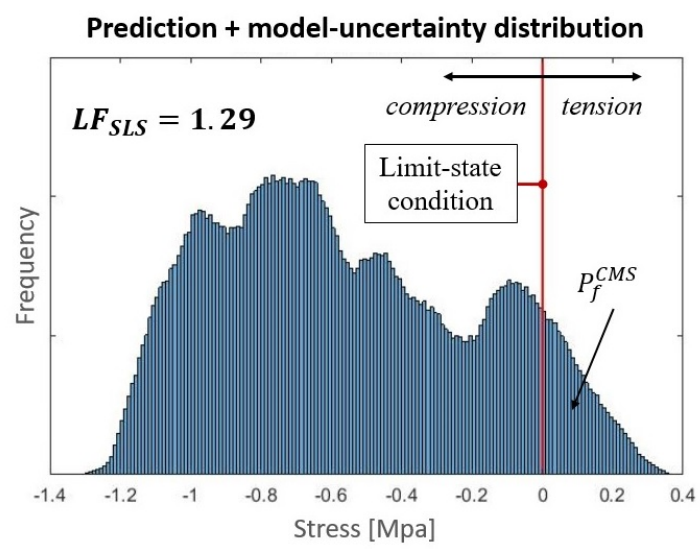

Fig. 9 CMS stress-predictions distribution including model uncertainty at failure (i.e. Load Factor=1.29) under the EC traffic loads. The SLS failure condition is shown using the red vertical line. The area on the right-hand side of the limit-state condition $P_{f}^{C M S}$ corresponds to the target failure probability $P_{f}^{S L S}$.

Table 7 RCap $_{S L S}$ assessments under the British code (BC) and the Eurocode (EC) according to analysis levels of detail.

\begin{tabular}{ccc}
\hline Analysis & BC traffic load & EC traffic load \\
\hline$S L S_{1}$ & 1.53 & 0.98 \\
$S L S_{2}$ & 1.82 & 1.42 \\
$S L S_{3}$ & 1.64 & 1.29 \\
\hline
\end{tabular}

\subsubsection{Sampling impact on reserve capacity}

In population-based approaches, the distribution of candidate-model predictions is relevant. Advanced sampling techniques such as radial-basis-function sampling (RFBS) are employed to increase the density of samples in candidate-model subdomains. This results in a denser CMS and more accurate distributions of candidate model predictions compared with traditional sampling approaches [39]. Therefore, methodologies for RCap assessment that involve only the worst-case model - instead of the candidate model distribution - do not exploit completely the information provided by falsification. In these circumstances, other identification techniques, such as constrained optimization, are more appropriate [50].

Table 8 reports RCap assessments using a range of sampling techniques and identification methodologies. In order to compare results with optimization approaches, no model uncertainties are added to model predictions, and only the worst-case scenario is considered (i.e. the worst prediction of the CMS).

Constrained optimization is the most conservative approach since parameter values are iteratively selected in order to provide the worst-case prediction with regards to the selected limit state function, while falsification thresholds are included as constraints. Taking advantage of advanced sampling techniques such as RBFS, the RCap assessed using EDMF is close to the worst-case scenario. Indeed, the thorough candidate-domain exploration results in accurate CMS predictions. Traditional sampling techniques, such as Latin hypercube sampling (LHS) may result in poor candidate-domain exploration and sparse CMS predictions. In these situations, CMS-prediction distributions may omit extreme predictions - which are 
Table 8 Impact of sampling in model identification on worst-case assessment of SLS reserve capacity.

\begin{tabular}{ccc}
\hline Identification methodology & Sampling technique & Reserve capacity for SLS \\
\hline EDMF & LHS & 1.38 \\
EDMF & RBFS & 1.26 \\
Constrained optimization & - & 1.25 \\
\hline
\end{tabular}

Table 9 Summary of ULS reserve-capacity estimations. Single-model analyses and population-based analyses are shown.

\begin{tabular}{llll}
\hline & Model / Analysis & Parameter values & RCap assessment \\
\hline$U L S_{1}$ & No FE model / design equations & Codes & Single model \\
$U L S_{2}$ & 2D FE model / non-linear & Codes & Single model \\
$U L S_{3}$ & 3D FE model / non-linear & Codes & Single model \\
$U L S_{4}$ & 2D FE model / non-linear & EDMF & Population based \\
$U L S_{5}$ & 3D FE model / non-linear & EDMF & Population based \\
\hline
\end{tabular}

essential to computing the failure probability. This results in an overestimation of the real structural resistance.

When the methodology described in Section 4.3.2.1 is used to compute the SLS RCap and the CMS probability of failure is compared with failure target probability, the RCap is equal to $1.29\left(S L S_{3}\right.$ in Table 7 ). This is less conservative than estimating the RCap using the worst-case scenario of identification and the partial-safety-factor method, which results in a RCap equal to 1.25. Further reference to the employment of constrained optimization for RCap estimations can be found in [50].

\subsection{Reserve capacity for ULS}

In this study, the constitutive law of concrete is assumed to be elastic perfectly-plastic with no tensile strength. In failures that are governed by shear mechanisms, the contribution of concrete tensile-strength is non-negligible [47]. Therefore, the assessment of ULS RCap is valid only when flexural failure of the bridge is more critical than shear failure.

Table 9 lists the ULS analyses that have been performed.

\subsubsection{Single-model methods $\left(U L S_{1,2,3}\right)$}

In this section, the ULS reserve capacity is assessed using a unique model that is defined by single parameter values which correspond to the design values.

\subsubsection{Simplified analysis $-U L S_{1}$}

In this analysis, only the most loaded beam of the bridge is evaluated and loads are estimated using the area-of-influence method (Figure 77. In $U L S_{1}$ design practices are used to estimate the RCap and parameter values are inferred from code specifications.

Figure 10 shows the mid-span-section flexural analysis that has been conducted to assess the bending capacity $\left(M_{R d}\right)$. The bending-action effect $\left(M_{E d}\right)$ is estimated using the simple model depicted in Figure 7 Finally, loads are increased by means of a load factor $(L F>1)$ until failure $\left(M_{E d}=M_{R d}\right)$. Load-factor values at the limit state are taken to be the ULS reserve capacity.

The $R C a p_{U L S}$ is equal to 1.68 under the $\mathrm{BC}$ traffic-load specifications and 1.05 under the EC traffic-load specifications. Therefore, more than $60 \%$ of the as-built ULS reserve 
capacity has been exploited following changes in traffic-load specifications between BC and EC. Since bridge assessment should employ state-of-the-art code requirements, in the remainder of this paper the RCap will be assessed considering only current traffic-load specifications [5].

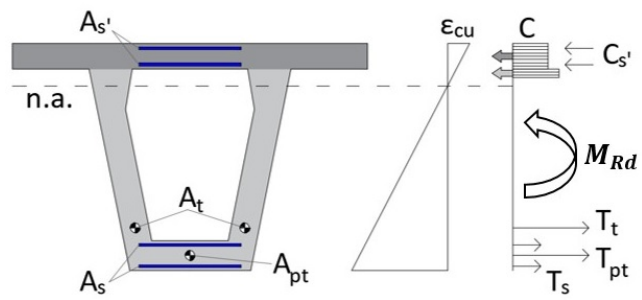

Fig. 10 Mid-span section analysis using design practices. Section geometry and area of reinforcements such as rebars in the deck $\left(A_{s}^{\prime}\right)$, rebars at the bottom $\left(A_{s}\right)$, tendons $\left(A_{t}\right)$ and pre-tensioning strands $\left(A_{p t}\right)$ are known. Assuming the maximum concrete strain equal to $\epsilon_{c u}$, the neutral-axis (n.a.) position is iteratively calculated until rotational equilibrium is satisfied. The bending moment at equilibrium indicates the section flexural capacity $\left(M_{R d}\right)$. Prestressing effects are included in the analysis.

\subsubsection{2D non-linear analysis - ULS $S_{2}$}

In $U L S_{2}$ a NLFEA is performed using a simplified 2D model that computes fast. Only the most loaded beam of the bridge is examined and loads are estimated as for the $U L S_{1}$ analysis.

A non-linear analysis based on elastic-plastic stress fields [28] is performed using the 2D model, which includes rebars and prestressing reinforcement, using the software JCONC [41] (Figure 11. Since JCONC does not support hollow-core sections, a $T$ section with equivalent inertia has been defined. This approximation has little impact on the stress distribution since the lower part of the section will be cracked at failure. Parameter values are taken from codes and loads are increased until failure (i.e. when concrete crushing is reached or computed solutions do not converge).

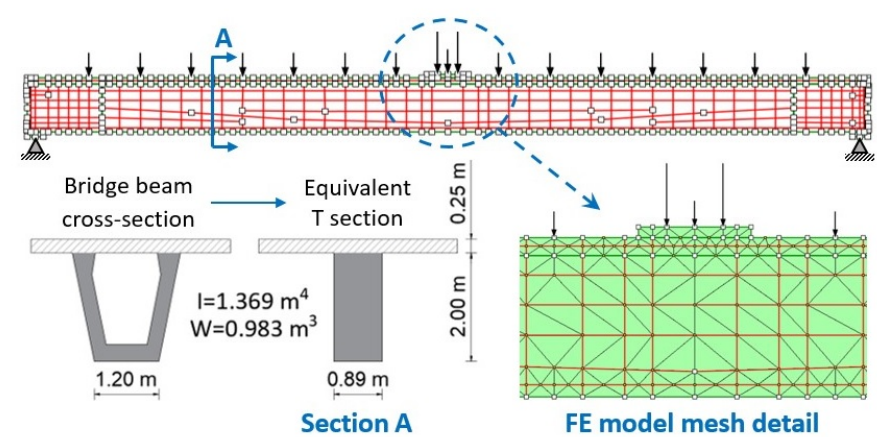

Fig. 11 2D FE model of the most-loaded beam (JCONC). The model includes rebars and prestressing reinforcement. Prestressing is introduced as an initial self-induced strain in the model. Since JCONC does not support hollow-core sections, a T section with equivalent inertia (I) has been defined. 
Figure 12 shows the effects of increasing loads in the results of the 2D NLFEA. At Stage $I$, which corresponds to the design loads for the ULS, the parabolic tendons are partially yielded (dark red areas), and high compressive stresses are observed in the deck (dark grey mesh elements). Then loads are gradually increased (Stage II), resulting in yielding of rebars and prestressing reinforcement. At Stage III the collapse is imminent. Compressive stress in the concrete deck at midspan reaches the maximum compressive strength (black mesh elements), and reinforcements are yielded at various locations. Further load increments depicted in Stage IV - result in structural failure of concrete and analysis solutions do not converge (see Figure 13 .

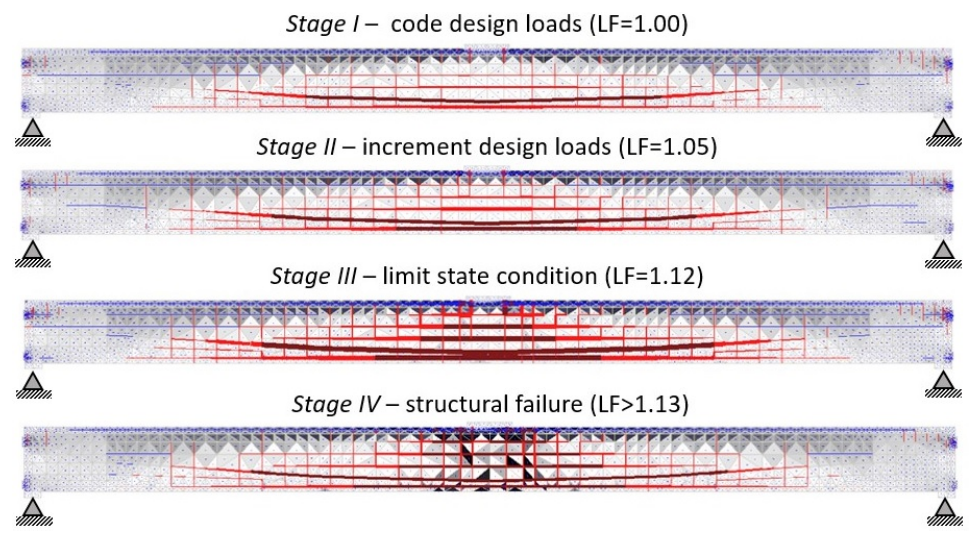

Fig. 12 Effects of increasing loads in the 2D non-linear finite-element analysis (NLFEA) performed using JCONC. The tension in rebars and prestressing reinforcement is shown in red. Yielding stress is shown in dark red. Grey-scaled mesh indicates concrete elements in compression, while concrete crushing is reached in black mesh-elements.

Figure 13 shows the solution error of NLFEA. As a rule, increasing the load factor (LF) results in greater solution errors. For LF higher than 1.13, there is no convergence, while load factors up to 1.12 provide errors in the range from 0.05 to 0.2 . According to JCONC specifications, solution errors lower than 0.45 at last iterations are deemed to be adequate. Therefore, the $R C a p_{U L S}$ is equal to 1.12 .

4.4.1.3 3D non-linear analysis - $U L S_{3}$

In $U L S_{3}$ the $3 \mathrm{D}$ model of the bridge is analysed (Figure 14). Parameter values are taken from codes and loads are increased until failure. This example involves the most advanced model of the structure; however, it requires long generation and computation times.

Figure 14 shows the 3D model where precast beams are simply supported. The stiffness of concrete barriers is neglected while their self-weight loading is applied to the deck. Reinforcement bars have not been modelled, while parabolic post-tensioning tendons and pre-tensioning strands are present. Concrete is modelled using a Drucker-Prager plasticity model. In order to improve convergence performance, prestress is modelled using equivalent loads and strengths of tendons and poured concrete are adjusted to account for biased stress conditions obtained after applying the equivalent loads.

Figure 15 shows the maximum bridge deflection with respect to the load factor applied to design loads. Yielding is observed first in tendons, and then in prestressing strands. Finally, 


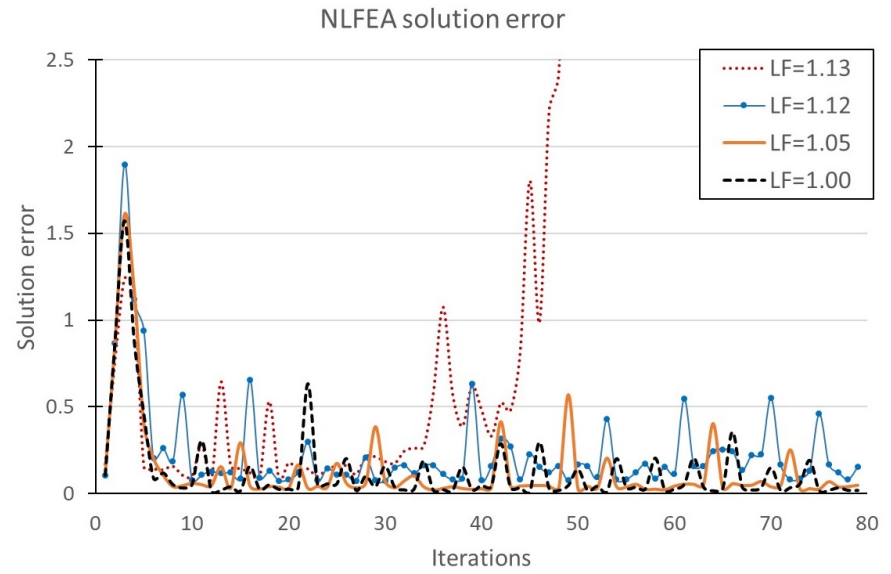

Fig. 13 Solution error of non-linear finite-element analysis (NLFEA). Increasing the load factor $(L F)$ the solution error increases. For $L F>1.13$ solutions do not converge; therefore, a $L F$ equal to 1.12 is the ULS reserve capacity in this case. Solution errors lower than 0.45 at last iterations are deemed to be adequate.

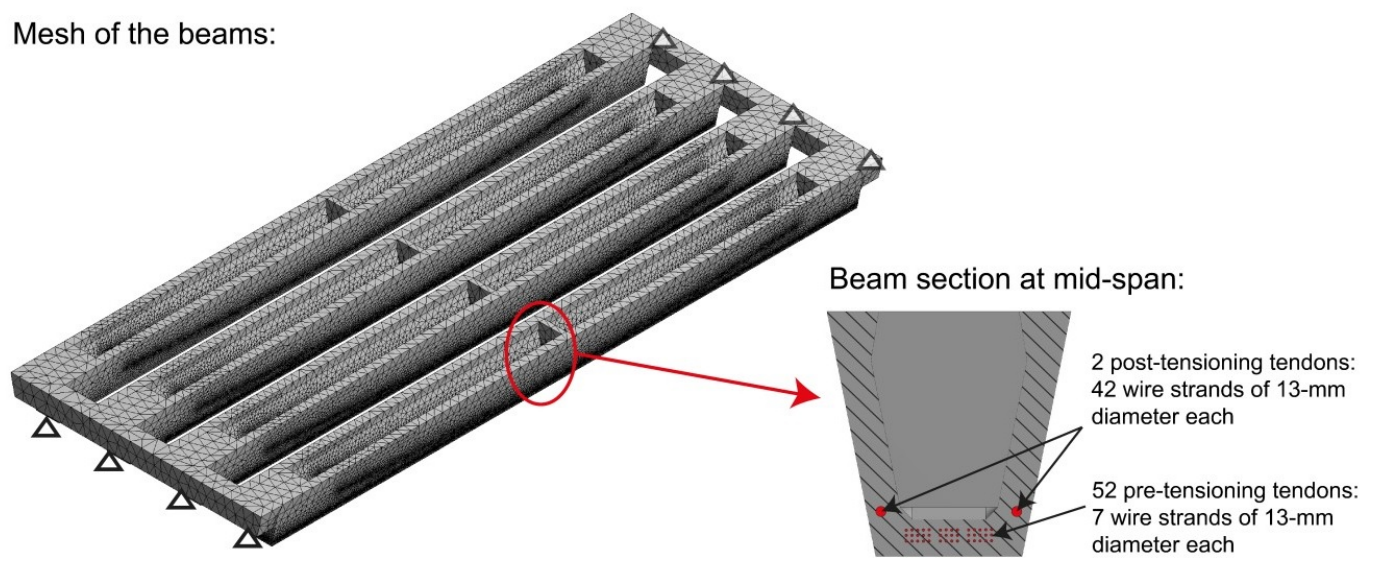

Fig. 14 Mesh and beam cross-section detail of the 3D model employed for NLFEA. Beams are simply supported, and prestressing reinforcement is modelled. The concrete deck is not displayed for clarity.

concrete crushing is reached in the deck and failure occurs. The $R \operatorname{Cap} p_{U S}$ is equal to 1.21.

\subsubsection{Population-based methods $\left(U L S_{4,5}\right)$}

In this section, the reserve capacity is assessed using the CMS. Table 10 reports active parameter values, which are taken from identification, along with additional parameter values, which are defined using design assumptions.

4.4.2.1 $2 D$ non-linear analysis $-U L S_{4}$

The same model as for the $U L S_{2}$ analysis is used. 


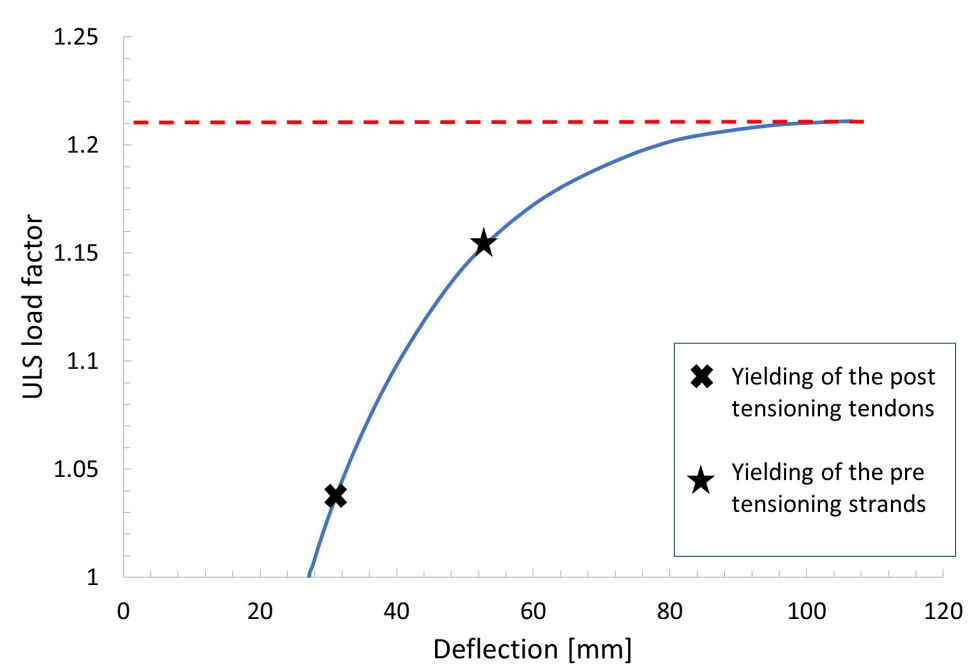

Fig. 15 Load factor with respect to the maximum bridge deflection.

Table 10 Active and additional parameters for the ULS. Design assumptions originate from drawings and the British Codes while parameter values are taken from the Eurocodes.

\begin{tabular}{llcc}
\hline Parameters & Status & Design assumption & ULS parameter values \\
\hline$\theta_{1}$ & Active & - & CMS distribution \\
$\theta_{2}$ & Active & - & CMS distribution \\
Cast-in-place concrete & Additional & BS 5400-4: grade 30/20 & $f_{c k}=25 \mathrm{MPa}$ \\
Precast concrete & Additional & BS 5400-4: grade 50/20 & $f_{c k}=40 \mathrm{MPa}$ \\
Rebars & Additional & BS 4449: grade 460B & $E=200 \mathrm{GPa} ; f_{y k}=460 \mathrm{MPa}$ \\
Prestressing tendons & Additional & BS 5896:1980 & $E=195 \mathrm{GPa} ; f_{p k}=1860 \mathrm{MPa}$ \\
\hline$E=$ Youngs modulus; $f_{c k}=$ concrete characteristic compressive strength; \\
$f_{y k}=$ steel characteristic tensile yield strength
\end{tabular}

This approach requires the calculation - using Equation (9) - of the global resistance safety factor $\gamma_{R}$ which includes NLFEA uncertainties (Equation (7)). However, estimating modelling uncertainty is a challenging task. The ECOV (estimate coefficient of variation) approach suggested in [47] provides a straightforward solution. In the ECOV approach, first the global safety factor $\gamma_{R}$ is computed according to Equation 9) without considering geometric and modelling uncertainties $\left(\nu_{M}=\nu_{G}=0\right)$, leading to a value of 1.19 . Then a modelling factor $\left(\gamma_{R d}\right)$ is included in the calculation of resistance according to the following equation:

$$
\operatorname{RCap}_{U S}=\frac{R_{m}}{\gamma_{R} \cdot \gamma_{R d}}
$$

where $R_{m}$ is equal to the reserve capacity (i.e. the load factor applied to the global vertical load at failure) when mean values of material properties are used. A modelling factor of $\gamma_{R d}=1.06$ is suggested for models with well-documented validity [8]. Using Equation (15), a $R C a p_{U L S}$ equal to 1.10 is obtained.

4.4.2.2 3D non-linear analysis - ULS $S_{5}$

The same model as for the $U L S_{3}$ analysis is used. 
Table 11 ULS reserve-capacity assessments using single-model methods ( $\left.U L S_{1,2,3}\right)$ and population-based methods $\left(U L S_{4,5}\right)$ under current traffic-load specifications.

\begin{tabular}{cc}
\hline Analysis & RCap $_{U L S}$ \\
\hline$U L S_{1}$ & 1.05 \\
$U L S_{2}$ & 1.12 \\
$U L S_{3}$ & 1.21 \\
$U L S_{4}$ & 1.10 \\
$U L S_{5}$ & 1.21 \\
\hline
\end{tabular}

First the global safety factor $\gamma_{R}=1.15$ is computed using 9 . Then, the ECOV approach is adopted considering a modelling factor, $\gamma_{R d}=1.06$, which leads to the assessment of a $R C a p_{U L S}$ equal to 1.21 .

If the BC traffic-load specification were employed in the $U L S_{5}$ analysis, the ULS RCap would be equal to 1.79 . Again, about $60 \%$ of the as-built reserve capacity has been exploited by adopting current-code traffic-load specifications (Eurocode with annexes for Singapore).

\subsubsection{Summary of ULS results}

RCap assessments for the ULS are summarised in Table 11. Advanced models based on NLFEA $\left(U L S_{2,3}\right)$ provide higher RCap values than simplified models $\left(U L S_{1}\right)$ when design values of parameters are employed, and uncertainties are included in the assessment using safety factors. The bridge has a ULS reserve capacity of approximately $20 \%$ using the Eurocode (including Singapore annexes).

When the global-safety-factor method is employed to assess RCap $\left(U L S_{4,5}\right)$, differences with models that employ parameter values taken from codes $\left(U L S_{3,4}\right)$ are not-significant in this case. Indeed, a similar value of modelling error is employed in the ECOV method and the partial safety factor method [43]. Furthermore, although some material properties (e.g. Youngs modulus) are inferred during identification, values of parameter that are more relevant for ultimate behaviour cannot be identified precisely. Values for those parameters could be updated through non-destructive testing and laboratory experiments. Such updating has not been performed in this case study.

\subsubsection{Sensitivity to modelling uncertainties}

Modelling-uncertainty magnitudes often exceed those associated with the partial-safetyfactor method. However, clear guidelines for uncertainty estimations are not available. Table 12 compares the assessment of RCap obtained considering a range of assumptions. In Case $I$ a single source of uncertainty (i.e. material uncertainty) is included since other uncertainties are reduced through model calibration and in-situ observations. Similar assumptions are made, for example, in [15]. Case II involves the ECOV method described above. Case III follows to the assumptions described in [43], which are reported in Table 13

Case I leads to a RCap assessment that is about $15 \%$ higher than the assessment in Case III. Moreover, the coefficients of variation $\nu_{F}$ computed using Equation (8) are much lower than those related to modelling and geometry uncertainties (see Table 12). Case III is the most conservative approach. In this situation, results do not provide significant advantages compared with simple calculations $\left(U L S_{1}\right)$. Accurate uncertainty estimation is crucial to provide trustworthy RCap assessments. Much literature has investigated NLFEA modelling uncertainties. For concrete structures under uniaxial bending, coefficients of variation in the range $(5 \%$ to $20 \%)$ are suggested in [43]. 
Table 12 Comparison of $R C a p_{U L S}$ assessments in population-based approaches considering three alternative assumptions for NLFEA uncertainties.

\begin{tabular}{cccccccc}
\hline \multicolumn{1}{c}{ Case I } & \multicolumn{2}{c}{ Case II } & \multicolumn{2}{c}{ Case III } \\
& $\nu_{F}$ & $\nu_{M}=\nu_{G}=0$ & $\operatorname{ECOV}\left(\gamma_{R d}=1.06\right)$ & $\nu_{M}=\nu_{G}=0.05$ \\
& & $\gamma_{R}$ & $R C a p_{U L S}$ & $\gamma_{R} \cdot \gamma_{R d}$ & $R C a p_{U L S}$ & $\gamma_{R}$ & $R C a p_{U L S}$ \\
$U L S_{4}$ & 0.038 & 1.12 & 1.17 & 1.19 & 1.10 & 1.28 & 1.03 \\
$U L S_{5}$ & 0.045 & 1.15 & 1.28 & 1.22 & 1.21 & 1.29 & 1.14 \\
\hline
\end{tabular}

Table 13 Coefficients of variation for log-normal distributed uncertainties of structural resistance (Case III).

\begin{tabular}{cc}
\hline Resistance uncertainties & Coefficient of variation \\
\hline $\mathrm{M}-$ modelling & $\nu_{M}=0.05$ \\
$\mathrm{G}-$ geometry & $\nu_{G}=0.05$ \\
$\mathrm{~F}-$ material & $\nu_{F} \Rightarrow$ Equation $[13$ \\
\hline
\end{tabular}

\section{Discussion}

The population-based methodologies described in this paper provide a rigorous approach to reserve-capacity assessment for structural identification. However, structural safety is ensured by subjective decisions, and this subjectivity often has the greatest impact on reliability assessments. Calculations of failure probabilities are linked to assumptions made during modelling and analysis. Therefore, similar values of the reliability index cannot be assumed to result in equivalent reserve-capacity estimations when several combinations of code specifications and analyses are employed. Reserve-capacity evaluations should employ state-of-the-art specifications and analyses; moreover, they must refer to requirements of current codes, particularly those that are developed specifically for the evaluation of existing structures. Nevertheless, in addition to providing indications of as-built reserve capacity, previously valid codes provide insight into the very conservative nature of design and construction practices when the bridge went into service.

This study introduces two data-interpretation methodologies to assess the reserve capacity of bridges according to SLS and ULS. Results for both limit states and the most advanced model classes (Table 14) indicate that worst case estimates of reserve capacity are $60 \%$ following design and construction and $20 \%$ following adoption of the Eurocode and including annexes for Singapore. Since these calculations use partial safety factors that have been intended for use during the design stage and since some modelling uncertainty is already included in partial safety factors, these values are lower bounds. Moreover, uncertainties in load effects are included using safety factors for traffic-load configurations that are given in codes. This ensures that values of reserve capacity are comparable and compliant with code requirements, which is crucial for asset managers. However, better estimates of real traffic loading would contribute significantly to increasing reserve-capacity estimations.

The estimation of uncertainties for non-linear analyses is a challenging task. Several authors [43 44] agree that further research is needed to recommend appropriate values of numerical-simulation uncertainties. Model validation techniques, as mentioned in [14], may provide useful input. Population-based methodologies that employ elastic measurements understandably provide limited advantages when non-linear finite-element analyses are used, since updated-parameter sensitivity is low and significant uncertainties remain. Future work will focus on the employment of non-destructive testing to update parameters that are relevant at the ULS. 
Table 14 Ultimate and serviceability reserve-capacity estimations. The worst case estimates using the most advanced model classes are reported in bold for each stage.

\begin{tabular}{cccc}
\hline Stage & Design traffic-load & RCap $_{S L S}$ & RCap $_{U L S}$ \\
& specifications & $S L S_{3}$ & $U L S_{5}$ \\
\hline Design and construction & British code & $\mathbf{1 . 6 4}$ & 1.79 \\
Assessment & Eurocode + SS annexes & 1.29 & $\mathbf{1 . 2 1}$ \\
\hline
\end{tabular}

\section{Conclusions}

Typical conservative practices carried out during design and construction have led to an asbuilt reserve capacity of $60 \%$. A large proportion of the as-built reserve capacity has been exploited to accommodate increased values of traffic-load specifications that are provided by current Singapore codes. However, the bridge still exhibits serviceability and ultimate reserve capacity - above all current safety factors - higher than $20 \%$. At the SLS, the proposed methodology takes advantage of the information obtained from prediction distributions of candidate models and helps avoid reserve-capacity overestimation due to inaccurate identification of the critical parameter-value combination. Also, precise sampling of parameter domains results in accurate extrapolations. At the ULS, the new approach allows estimation of the reserve capacity through limiting the number of time-consuming non-linear analyses, while advanced models have helped exploit reserve sources that are neglected by conservative models.

Acknowledgements The research was conducted at the Future Cities Laboratory at the Singapore-ETH Centre, which was established collaboratively between ETH Zurich and Singapore's National Research Foundation (FI 370074016) under its Campus for Research Excellence and Technological Enterprise programme. The authors gratefully acknowledge the support of the Land Transport Authority (LTA) of Singapore for support during load tests in the scope of the case study.

\section{Conflict of Interest:}

The authors declare that they have no conflict of interest.

\section{References}

1. Bs-5400-2:1978 steel, concrete and composite bridges. Specification for loads (1978)

2. Bs-5400-4:1984 steel, concrete and composite bridges. Code of practice for design of concrete bridges (1984)

3. En 1990 eurocode 0 - basis of structural design (2002)

4. Sia-269:2011 existing structures - Bases (2011)

5. Ss-en-1991-2 eurocode 1 actions on structures - Part 2: Traffic loads on bridges (2012)

6. Ss-en-1992-2 eurocode 2 design of concrete structures - Concrete bridges: Design and detailing rules (2012)

7. Beck, J.L., Katafygiotis, L.S.: Updating models and their uncertainties. I: Bayesian statistical framework. Journal of Engineering Mechanics 124(4), 455-461 (1998)

8. Blomfors, M., Engen, M., Plos, M.: Evaluation of safety formats for non-linear finite element analyses of statically indeterminate concrete structures subjected to different load paths. Structural Concrete 17(1), 44-51 (2016)

9. Brhwiler, E., Vogel, T., Lang, T., Lchinger, P.: Swiss standards for existing structures. Structural Engineering International 22(2), 275-280 (2012) 
10. Brownjohn, J.M.W., De Stefano, A., Xu, Y.L., Wenzel, H., Aktan, A.E.: Vibration-based monitoring of civil infrastructure: challenges and successes. Journal of Civil Structural Health Monitoring 1(3), 79-95 (2011)

11. Catbas, F., Kijewski-Correa, T., Lynn, T., Aktan, A.E., others: Structural identification of constructed systems. American Society of Civil Engineers, (2013)

12. Catbas, F.N., Aktan, A.E.: Condition and damage assessment: issues and some promising indices. Journal of Structural Engineering 128(8), 1026-1036 (2002)

13. Cervenka, V.: Computer simulation of failure of concrete structures for practice. In: 1st fib Congress, pp. 289-304 (2002)

14. Cervenka, V.: Reliability-based non-linear analysis according to fib Model Code 2010. Structural Concrete 14(1), 19-28 (2013)

15. Cervenka, V., Cervenka, J., Pukl, R.: Safety assessment in fracture analysis of concrete structures. In: Proceedings of the 6th International Conference on Fracture Mechanics of Concrete and Concrete Stuctures, Catania, vol. 2, pp. 1043-1049 (2007)

16. Frangopol, D., Tsompanakis, Y.: Maintenance and safety of aging infrastructure: Structures and infrastructures book series, vol. 10. CRC press (2014)

17. Frangopol, D.M.: Life-cycle performance, management, and optimisation of structural systems under uncertainty: accomplishments and challenges 1. Structure and Infrastructure Engineering 7(6), 389-413 (2011)

18. Ghosn, M., Frangopol, D.M., McAllister, T.P., Shah, M., Diniz, S.M.C., Ellingwood, B.R., Manuel, L., Biondini, F., Catbas, N., Strauss, A., others: Reliability-based performance indicators for structural members. Journal of Structural Engineering 142(9), F4016002 (2016)

19. Goller, B., Beck, J.L., Schueller, G.I.: Evidence-based identification of weighting factors in Bayesian model updating using modal data. Journal of Engineering Mechanics 138(5), 430-440 (2011)

20. Goulet, J.A., Kripakaran, P., Smith, I.F.C.: Multimodel structural performance monitoring. Journal of Structural Engineering 136(10), 1309-1318 (2010)

21. Goulet, J.A., Smith, I.F.C.: Structural identification with systematic errors and unknown uncertainty dependencies. Computers \& structures 128, 251-258 (2013)

22. Helland, S.: Design for service life: implementation of fib Model Code 2010 rules in the operational code ISO 16204. Structural Concrete 14(1), 10-18 (2013)

23. Holick, M., Nvarov, V., Gottfried, R., Kronika, M.: Basics for assessment of existing structures. eds (2014)

24. Lam, H.F., Yang, J., Au, S.K.: Bayesian model updating of a coupled-slab system using field test data utilizing an enhanced Markov chain Monte Carlo simulation algorithm. Engineering Structures 102, 144-155 (2015)

25. Lounis, Z., McAllister, T.P.: Risk-based decision making for sustainable and resilient infrastructure systems. Journal of Structural Engineering 142(9), F4016005 (2016)

26. Miller, R.A., Aktan, A.E., Shahrooz, B.M.: Destructive testing of decommissioned concrete slab bridge. Journal of Structural Engineering 120(7), 2176-2198 (1994)

27. Minervino, C., Sivakumar, B., Moses, F., Mertz, D., Edberg, W.: New AASHTO guide manual for load and resistance factor rating of highway bridges. Journal of Bridge Engineering 9(1), 43-54 (2004)

28. Muttoni, A., Fernndez Ruiz, M., Niketic, F., Backes, M.R.: Assessment of existing structures based on elastic-plastic stress fields-Modelling of critical details and investigation of the in-plane shear transverse bending interaction. Tech. rep., Rapport OFROU, N. 680, Switzerland (2016)

29. Muttoni, A., Ruiz, M.F.: Levels-of-A pproximation Approach in Codes of Practice. Structural Engineering International 22(2), 190-194 (2012)

30. Neves, A.C., González, I., Leander, J., Karoumi, R.: Structural health monitoring of bridges: a model-free ann-based approach to damage detection. Journal of Civil Structural Health Monitoring 7(5), 689-702 (2017)

31. Pasquier, R., DAngelo, L., Goulet, J.A., Acevedo, C., Nussbaumer, A., Smith, I.F.C.: Measurement, data interpretation, and uncertainty propagation for fatigue assessments of structures. Journal of Bridge Engineering 21(5), 04015087 (2016)

32. Pasquier, R., Goulet, J.A., Acevedo, C., Smith, I.F.C.: Improving fatigue evaluations of structures using in-service behavior measurement data. Journal of Bridge Engineering 19(11), 04014045 (2014)

33. Pasquier, R., Smith, I.F.C.: Robust system identification and model predictions in the presence of systematic uncertainty. Advanced Engineering Informatics 29(4), 1096-1109 (2015)

34. Pasquier, R., Smith, I.F.C.: Sources and forms of modelling uncertainties for structural identification. In: 7th International Conference on Structural Health Monitoring of Intelligent Infrastructure (SHMII) (2015)

35. Pasquier, R., Smith, I.F.C.: Iterative structural identification framework for evaluation of existing structures. Engineering Structures 106, 179-194 (2016) 
36. Pimentel, M., Brhwiler, E., Figueiras, J.: Safety examination of existing concrete structures using the global resistance safety factor concept. Engineering Structures 70, 130-143 (2014)

37. Pimentel, M., Brwhiler, E., Figueiras, J.: Extended cracked membrane model for the analysis of RC panels. Engineering Structures 32(8), 1964-1975 (2010)

38. Plos, M., Shu, J., Zandi, K., Lundgren, K.: A multi-level structural assessment strategy for reinforced concrete bridge deck slabs. Structure and Infrastructure Engineering 13(2), 223-241 (2017)

39. Proverbio, M., Costa, A., Smith, I. F. C.: Adaptive sampling methodology for structural identification using radial-basis functions. Journal of Computing in Civil Engineering 32(3), 04018008 (2018)

40. Richard, B., Epaillard, S., Cremona, C., Elfgren, L., Adelaide, L.: Nonlinear finite element analysis of a 50 years old reinforced concrete trough bridge. Engineering Structures 32(12), 3899-3910 (2010)

41. Ruiz, M.F., Muttoni, A.: On development of suitable stress fields for structural concrete. ACI Structural journal 104(4), 495 (2007)

42. Schlune, H., Plos, M., Gylltoft, K.: Improved bridge evaluation through finite element model updating using static and dynamic measurements. Engineering structures 31(7), 1477-1485 (2009)

43. Schlune, H., Plos, M., Gylltoft, K.: Safety formats for non-linear analysis of concrete structures. Magazine of Concrete Research 64(7), 563-574 (2012)

44. Schneider, J.: Introduction to safety and reliability of structures, vol. 5. Iabse (2006)

45. Simoen, E., Papadimitriou, C., Lombaert, G.: On prediction error correlation in Bayesian model updating. Journal of Sound and Vibration 332(18), 4136-4152 (2013)

46. Smith, I.F.C.: Studies of Sensor Data interpretation for Asset Management of the Built environment. Frontiers in Built Environment 2, 8 (2016)

47. fib International Federation for Structural Concrete: Model Code for concrete structures 2010 (2010)

48. Tapan, M., Aboutaha, R.S.: Strength evaluation of deteriorated RC bridge columns. Journal of Bridge Engineering 13(3), 226-236 (2008)

49. of Transportation, U.D., Administration, F.H.: 2015 Status of the Nations Highways, Bridges and Transit: Conditions and Performance (2017)

50. Vernay, D., Favre, F.X., Smith, I.F.C.: Robust model-updating methodology for estimating worst-case loading capacity of existing bridges. Journal of Civil Structural Health Monitoring p. Under review

51. Vrouwenvelder, T.: The JCSS probabilistic model code. Structural Safety 19(3), 245-251 (1997)

52. Winiewski, D.F., Casas, J.R., Ghosn, M.: Codes for safety assessment of existing bridgescurrent state and further development. Structural Engineering International 22(4), 552-561 (2012)

53. World Economic Forum: Strategic infrastructure, steps to operate and maintain infrastructure efficiently and effectively. Tech. Rep. 180314, World Economic Forum, Davos (2014)

54. Zheng, Y., Robinson, D., Taylor, S., Cleland, D.: Finite element investigation of the structural behaviour of deck slabs in composite bridges. Engineering Structures 31(8), 1762-1776 (2009) 